\title{
On secure system performance over SISO, MISO and MIMO-NOMA wireless networks equipped a multiple antenna based on TAS protocol
}

\author{
Thanh-Nam Tran* (D) and Miroslav Voznak
}

\begin{abstract}
This study examined how to improve system performance by equipping multiple antennae at a base station (BS) and all terminal users/mobile devices instead of a single antenna as in previous studies. Experimental investigations based on three NOMA down-link models involved (1) a single-input-single-output (SISO) scenario in which a single antenna was equipped at a BS and for all users, (2) a multi-input-single-output (MISO) scenario in which multiple transmitter antennae were equipped at a BS and a single receiver antenna for all users and (3) a multi-input-multi-output (MIMO) scenario in which multiple transmitter antennae were equipped at a BS and multiple receiver antenna for all users. This study investigated and compared the outage probability (OP) and system throughput assuming all users were over Rayleigh fading channels. The individual scenarios also each had an eavesdropper. Secure system performance of the individual scenarios was therefore also investigated. In order to detect data from superimposed signals, successive interference cancellation (SIC) was deployed for users, taking into account perfect, imperfect and fully imperfect SICs. The results of analysis of users in these three scenarios were obtained in an approximate closed form by using the Gaussian-Chebyshev quadrature method. However, the clearly and accurately presented results obtained using Monte Carlo simulations prove and verify that the MIMO-NOMA scenario equipped with multiple antennae significantly improved system performance.
\end{abstract}

Keywords: Single-input-single-output (SISO), Multi-input-single-output (MISO), Multi-input-multi-output (MIMO), Non-orthogonal multiple access (NOMA), Transmitter antenna selection (TAS), Secrecy outage probability (SOP), Imperfect SIC

\section{Introduction}

The explosive growth of mobile devices and the Internet of Things (IoT) is facing a trend of increased wireless network traffic in future networks. Researchers have confirmed non-orthogonal multiple access (NOMA) as the candidate to become the fifth generation (5G) wireless communication technology [1-4]. Liu et al. [5] demonstrated that the NOMA system has a better ergodic sum rate (ESR) than the orthogonal multiple access (OMA) system. The key technologies of NOMA are lower latency, enhanced fairness between users and a better efficiency

*Correspondence: thanh.nam.tran.st@vsb.cz

Faculty of Electrical Engineering and Computer Science, Technical University of Ostrava, 17. listopadu 2172/15, 70833 Ostrava-Poruba, Czech Republic spectrum, because all user equipment (UE) is served in the same time slot or frequency by sharing the spectrum with different allocation power coefficients based on the UE channel conditions in the same power domain. The base station (BS) sends a superimposed signal to all UE in the same time slot. For example, the downlink NOMA system consists of nearby UE with strong channel conditions and distant UE with poor channel conditions [6-10]. At the UE, the signals received are decoded by applying successive interference cancellation (SIC) until their own information is successfully detected $[11,12]$. For example, the nearby UE decodes the data symbol of the distant UE first and then decodes its own data symbol after subtracting the decoded data symbol of the distant UE. In addition, the distant UE only 
decodes its own data symbol by treating the nearby UE data symbol as noise. In [6], the authors studied the outage probability (OP) and ergodic sum capacity (ESC) of NOMA systems with randomly distributed UE in the neighbourhood of the $\mathrm{BS}$ and verified that the performance of a NOMA system considerably outperformed an OMA system when the allocation power scheme was deployed.

In order to improve system performance, researchers have proposed many different technologies. One of them is cooperative communications, which deploys relays as an effective solution in order to combat fading. The authors studied full-duplex (FD) relays to avoid wasting time slot/frequency by replacing half-duplex (HD) relays [13]. The authors also proposed using $N-1 \mathrm{FD}$ relays to support the $N$ th users with the poorest channel conditions [14]. The authors indicated that system performance could be enhanced by increasing the $m$ coefficient of the Nakagami- $m$ fading channels compared to the Rayleigh fading channels. As expected, in accordance with capability and reality, some wireless technologies combined with NOMA were proposed in order to scale up system performance: cooperative communication [15, 16], full duplex [17], cognitive radio (CR) [18, 19], millimetre wave [20], visible light communication [21], etc.

Other, different protocols, such as HD, FD, decode-andforward (DF) and amplify-and-forward (AF) with fixed gain (FG) or variable gain (VG), were also studied in order to find a better protocol to implement with NOMA technology. In [22], the DF protocol was deployed. The advantage of DF protocol is forwarded signals without including the data symbols of the previous UE and simplicity in analysis and simulation. The authors also considered deploying AF protocol with fixed gain (FG) or variable gain (VG). However, the authors demonstrated that $\mathrm{DF}$ protocol is better than AF protocol depending on certain parameters, and conversely, AF protocol is better than DF with other parameters. The authors therefore proposed a mechanism for switching adaptive protocols according the parameters in order to optimize system performance.

However, previous studies have commonly assumed that only a single antenna was equipped on network nodes. Recently, researchers have proposed multiple antenna technology as a powerful option for enhancing system performance [23-26]. The authors investigated the system performance of a NOMA network with multiple antennae and an energy harvesting $(\mathrm{EH})$ relay on the OP performance [27]. Although the system performance can be potentially improved by equipping more antennae, the improvement is limited by the cost of radio frequency (RF) technology at the UE. In order to avoid expensive hardware costs and keep the throughput profits from multiple antennae, a transmit antenna selection (TAS) protocol was verified and admitted as a powerful option [28]. In [5], the authors investigated OP in a dual-hop relay over a MIMO-NOMA network with TAS and maximum ratio combining (MRC) protocols over the Rayleigh fading channels. In the results of the study, the authors recognized that the system performance could be improved increasing the number of antennae.

On the other hand, physical layer security (PLS) is a topic popular not only in wireless communications but also network security. PLS can see secret communications by exploiting the entropy and confuse time of the wireless channels without the use of an encoding algorithm [29]. Zhang et al. investigated the secrecy system performance of a SISO-NOMA system and verified the secrecy sum rate of a NOMA system as superior to a traditional OMA system [30]. The authors investigated the PLS of NOMA systems in massive networks where all UE and eavesdroppers were located at random positions [31] and obtained new, precise asymptotic expressions for secrecy outage probability (SOP) [32]. The authors in [33] assumed that the BS had full channel state information (CSI) in both the main channels of trusted UE and the wiretap channels of non-trusted UE and proposed optimal antenna selection (OAS) and sub-optimal antenna selection (SAS) protocol schemes in order to improve the secrecy performance of a MIMO system compared to an ordinary space-time transmission (STT) protocol. Precise asymptotic expressions in closed form for the SOP of a MIMO system with underlay was obtained in [34]. The results indicated that both SAS and OAS protocols could considerably improve secrecy performance. In [35], Lei et al. investigated the secrecy performance of two types of UE over down-link MOMA systems in which SISO and MISO schemes were applied with different TAS methods. However, the authors have assumed that the UE only had one receiver antenna. From previously studied results, we are considering an investigations of non-secrecy outage probability (NOP) and SOP of UE over three NOMA schemes SISO, MISO and MIMO systems with TAS protocol as motivations.

Some important and recent studies similar to this research are [36-43]. In the excellent work [36], the authors investigated SOP for the cooperative NOMA (CNOMA) in CR networks and took into account the impact of distance of users from the BS on secrecy performance. Although the authors investigated CR-NOMA with multiple users and multiple eavesdroppers, the power allocation (PA) coefficients for users were fixed. Our objective is to implement a PA strategy to ensure QoS for users. Zhou et al. [37] improved the secrecy performance of NOMA system based on CR networks using simultaneous wireless information and power transfer (SWIPT). Another work [38] investigated the popular PLS topic in order to find a way to minimize power over MISO-NOMA 
systems. In the work in [39], the authors fully surveyed the special issues in PLS, such as PLS fundamentals, C-NOMA for PLS, cooperative jamming for PLS and hybrid C-NOMA for PLS. In another work [40], the authors investigated the secrecy performance of random MIMO-NOMA with homogeneous Poisson point processes (HPPPs) on both the BS and users over $\alpha-\mu$ fading channels. The authors obtained analysis results and verified them with Monte Carlo simulation results. From the obtained results, the author indicated that SOP performance was impacted by the number of users, the path-loss exponent and the number of antennae. In the work [41], the authors investigated a MIMO-NOMA system based on TAS protocol for two users over Nakagami- $m$ fading channels. Although the work was interesting, the authors, however, did not consider the PA issue, such as in [36]. Feng et al. [42] considered PA issue in order to maximize the QoS for strong user while guaranteeing the QoS for weak user. In another work [43], the authors investigated two source-destination pairs through two-stage secure relay selection (TSSRS) in order to maximize the SOP of one source-destination pair, while guaranteeing the SOP of the other pair. The author, however, only equipped a single antenna for all source, relay, destination, and eavesdropper.

The featured study investigated certain issues that form its primary contribution:

- This paper investigated and compared the system performance in different scenarios of SISO, MISO and MIMO architecture with deploying TAS protocol in order to determine which had the best quality of service (QoS) for users. Although the investigations were based on two users, this study can be extended to $N$ users, such as in [14].

- This paper investigated system performance on various criteria such as non-secure outage probability (NOP), secure outage probability (SOP) and system throughput. Novel closed forms and approximate forms were obtained from these investigations.

- This paper also investigated the impact of antennae on system performance. The impact of perfect/imperfect/fully imperfect SIC were also considered.

- Finally, the analysis results were demonstrated and verified with Monte Carlo simulation results.

This study was presented in the following structure. In experimental model section, three models, SISO, MISO and MIMO-NOMA, considering imperfect SIC, respectively, are proposed and analysed. In the next section, system performance is analysed and the closed form expressions based on NOP, SOP and system throughput are obtained. In Section 4, the study proposes the system parameters for investigations and simulations using
Monte Carlo simulations in Matlab software ${ }^{1}$. The results are presented in the figures. A detailed discussion based on the obtained results is given as figures. Finally, a summary of the study's results is presented in the "Conclusion" section.

This paper uses some notations such as

[.] referred the matrix.

$\max \{$.$\} referred the maximum function.$

$\operatorname{Pr}\{$.$\} referred the probability.$

$E\{$.$\} referred the mean function.$

$f_{(.)}(x)$ referred probability density function (PDF).

$F_{(.)}(x)$ referred cumulative distribution function (CDF).

\section{Experimental models}

This study investigated the system performance on NOP and SOP of two types of UE over three individual downlink scenarios: (1) SISO, (2) MISO and (3) MIMO-NOMA with the TAS protocol.

The system model proposed is shown in Figure 1. Two users wait for serving signals from the BS. The BS sends a superimposed signal $\vartheta=\sqrt{P_{0}}\left(\sqrt{\alpha_{1}} x_{1}+\sqrt{\alpha_{2}} x_{2}\right)$ to both $U_{1}$ and $U_{2}$ in the same time slot and the same power domain, where $\alpha_{1}$ and $\alpha_{2}$ are the allocation power coefficients of the users $U_{1}$ and $U_{2}$, respectively. According to the terms of NOMA theory, user $U_{2}$ with poor channel conditions was prioritized to allocate a larger power coefficient than user $U_{2}$, whereas $\alpha_{1}<\alpha_{2}$ and $\alpha_{1}+\alpha_{2}=1$.

As a feature study, Ding et al. [6] proposed a down-link NOMA system with random $\chi$ users and proposed a PA strategy for random $\chi$ users as $\alpha_{i}^{[6]}=\frac{\chi-i+1}{1+\cdots+\chi}$ for $i \in \chi$, where $U_{1}$ to $U_{\chi}$ were the poorest user to the strongest user. But $U_{1}$ to $U_{\chi}$ in our model were the strongest user to the poorest user. We therefore proposed a Dings' modified PA strategy as $\alpha_{i}^{[6]}=\frac{i}{1+\cdots+\chi}$. To simplify, for this study, we assumed $\chi=2$. We realized, however, that this PA strategy fixed a PA coefficient depending on the number of $\chi$ users. For example, $\chi=2$, we obtained the PA coefficients as $\alpha_{1}^{[6]}=0.3333$ and $\alpha_{2}^{[6]}=0.6667$ without considering the strong channel conditions or slight differences between the two users. Tran et al. [14] proposed the PA strategy for strongest user and poorest user as $\alpha_{i}^{[14]}=\sigma_{0, \chi-i+1}^{2} / \sum_{k=1}^{\chi} \sigma_{0, k}^{2}$. This study therefore respectively obtained the PA factors for $U_{1}$ and $U_{2}$ as $\alpha_{1}^{[14]}=\sigma_{0,2}^{2} / \sum_{k=1}^{2} \sigma_{0, k}^{2}$ and $\alpha_{2}^{[14]}=\sigma_{0,1}^{2} / \sum_{k=1}^{2} \sigma_{0, k}^{2}$ with assuming the BS own fully CSIs. We investigated and compared both of these PA strategies as a contribution.

\subsection{SISO scenario}

A common scenario in previous studies, such as $[6,14]$, the BS and users were equipped only with a sin-

\footnotetext{
${ }^{1}$ This study used Matlab software version R2017b made by The MathWorks,
} Inc. based at 3 Apple Hill Drive Natick, MA 01760 USA 508-647-7000. 


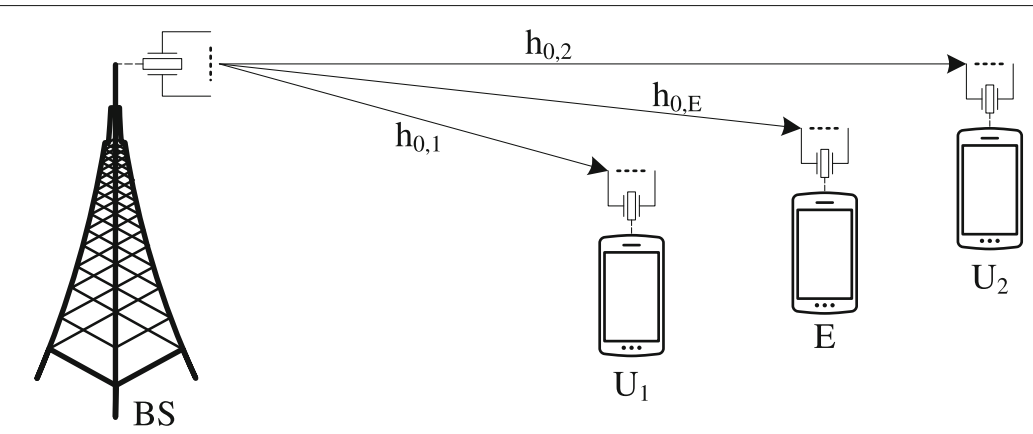

(a)

\begin{tabular}{|c|c|c|}
\hline$U_{1}$ & $U_{i}(i \in \chi)$ & $U_{\chi}$ \\
\hline$\alpha_{1}=\frac{1}{1+\cdots+\chi}$ & $\alpha_{i}=\frac{i}{1+\cdots+\chi}$ & $\alpha_{\chi}=\frac{\chi}{1+\cdots+\chi}$ \\
\hline
\end{tabular}

(b)

\begin{tabular}{|c|c|c|}
\hline$U_{1}$ & $U_{i}(i \in \chi)$ & $U_{\chi}$ \\
\hline$\alpha_{1}=\frac{\sigma_{0, \chi}^{2}}{\sigma_{0,1}^{2}+\cdots+\sigma_{0, \chi}^{2}}$ & $\alpha_{1}=\frac{\sigma_{0, \chi-i+1}^{2}}{\sigma_{0,1}^{2}+\cdots+\sigma_{0, z}^{2}}$ & $\alpha_{\chi}=\frac{\sigma_{0,1}^{2}}{\sigma_{0,1}^{2}+\cdots+\sigma_{0, \chi}^{2}}$ \\
\hline
\end{tabular}

(c)

Fig. 1 a MIMO-NOMA wireless network with $\chi=2$ users, and the PA coefficient for ith user whereas $i \in \chi \mathbf{b}$ like [6], and $\mathbf{c l i k e ~ [ 1 4 ] ~}$

gle antenna. Therefore, a single connection from the BS to each user was denoted by $h_{0, i}^{(1,1)}$ for $i=\{1,2, E\}$ where the channel $h_{0, i}^{(1,1)}$ followed $h_{0, i}^{(1,1)}=d_{0, i}^{-r}$, where $d_{0, i}$ refers to the distance from the $\mathrm{BS}$ to $U_{i}$ and $r$ refers the path-loss exponent factor [44]. This study assumed all users were over Rayleigh fading channels.

The received signals at both $U_{1}$ and $U_{2}$ were respectively expressed as follows:

$$
y_{i}^{(\mathrm{SISO})}=y_{i}^{(1,1)}=h_{0, i}^{(1,1)} \sqrt{P_{0}}\left(\Lambda \sqrt{\alpha_{1}} x_{1}+\Delta \sqrt{\alpha_{2}} x_{2}\right)+n_{i},
$$

where $P_{0}$ refers to the BS's transmission power and the subsequent signal-to-noise-ratio (SNR) $\rho_{0}=P_{0} / N_{0}$, and $n_{i}$ for $i=\{1,2, E\}$ refers to additive white Gaussian noises (AWGNs) followed by $n_{i} \sim C N\left(0, N_{0}\right)$ with zero mean and variance $N_{0}$.

By deploying the SIC as [43] after reversing user arrangement, the user $U_{i}$ obtains the signal-tointerference-plus-noise ratios (SINRs) when it decodes $x_{j}$ symbol as follows:

$$
\begin{aligned}
& \gamma_{i-x_{j}}^{(\mathrm{SISO})}=\gamma_{i-x_{j}}^{(1,1)} \stackrel{(j=2)}{=} \frac{\Delta^{2}\left|h_{0, i}^{(1,1)}\right|^{2} \alpha_{2} P_{0}}{\Lambda^{2}\left|h_{0, i}^{(1,1)}\right|^{2} \alpha_{1} P_{0}+N_{0}}, \\
& \stackrel{(j=1)}{=}\left(\Lambda^{2}\left|h_{0, i}^{(1,1)}\right|^{2} \alpha_{1} P_{0}\right) / N_{0},
\end{aligned}
$$

where $i=\{1,2\}$ and $j=\{2,1\}$. There are two SIC phases at $U_{1}$. The first SIC phase obtains the SINR as (2) for $i=1$ and $j=2$ when $U_{1}$ decodes $U_{2} \mathrm{~s}^{\prime} x_{2}$ symbol and removes $x_{2}$ symbol from the received signal. $U_{1}$ then decodes its own $x_{1}$ symbol and obtains SINS as (3) for $i=j=1$ at the second SIC phase. On another hand, it is important to note that the user $U_{2}$ only detects its own $x_{2}$ symbol by treating $x_{1}$ symbol as noise and obtains the SINR as (3) for $i=j=2$. In addition, this paper assumed that the users deployed imperfect SIC [45] denoted by coefficients $0 \leq$ $\Lambda^{2} \leq 1$ and $0 \leq \Delta^{2} \leq 1$. For clarity, when $U_{2}$ detects its own $x_{2}$ symbol by treating $x_{1}$ as interference, then $\Lambda^{2}=0$ refers to perfect SIC, $\Lambda^{2}=1$ refers to fully imperfect SIC, and otherwise referred imperfect SIC.

The instantaneous bit rate of $U_{i}$ is obtained when it detects $x_{j}$ symbol expressed as follows: 


$$
R_{i-x_{j}}^{(\mathrm{SISO})}=R_{i-x_{j}}^{(1,1)}=\log _{2}\left(1+\gamma_{i-x_{j}}^{(1,1)}\right)
$$

where $i=\{1,2, E\}$ and $j=\{2,1\}$.

\subsection{MISO scenario}

Previous research results have indicated that system performance improved by equipping more antennae. We in this subsection assumed that BS was equipped a multiple transmitter antenna, denoted by $S$ for $S>1$ and followed by matrix channel as $\mathbf{H}_{0, i}^{(\mathrm{MISO})}=\left[h_{0, i}^{(1,1)} \cdots h_{0, i}^{(s, 1)} \cdots h_{0, i}^{(S, 1)}\right]$ for $s \in S$. Vector transmitter antennae on BS send a broadcast beamforming superimposed signal to all users as in [46].

Therefore, the vector beamforming received signals from $S$ antennae on the BS to the user $U_{i}$ for $i=\{1,2, E\}$ are expressed as follows:

$$
\begin{aligned}
y_{i}^{(\mathrm{MISO})} & =\operatorname{matrix}_{s=[1 \cdots S]}\left[y_{i}^{(s, 1)}\right] \\
& =\operatorname{matrix}_{s=[1 \cdots S]}\left[h_{0, i}^{(s, 1)}\right]\left(\Lambda \sqrt{\alpha_{1} P_{0}} x_{1}+\Delta \sqrt{\alpha_{2} P_{0}} x_{2}\right)+n_{i},
\end{aligned}
$$

where $h_{0, i}^{(s, 1)} \in \mathbf{H}_{0, i}^{(\mathrm{MISO})}$ refers the channel from the $s$ th transmitter antenna for vector $s=[1 \cdots S]$ on BS to the receiver antenna on $U_{i}$ for $i=\{1,2, E\}$.

The TAS protocol in this subsection deployed. The user $U_{i}$ for $i=\{1,2, E\}$ therefore obtains SINRs when it detects $x_{j}$ symbol for $j=\{2,1\}$ with implementing TAS protocol expressed as follows:

$$
\begin{aligned}
\gamma_{i-x_{j}}^{(\text {MISO })}= & \max _{s=[1 \cdots S]}\left\{\left[\gamma_{i-x_{j}}^{(s, 1)}\right]\right\} \\
& \stackrel{(j=2)}{=} \frac{\max \left\{\left[\left|h_{0, i}^{s, 1}\right|^{2}\right]\right\} \Delta^{2} \alpha_{2} P_{0}}{\max \left\{\left[\left|h_{0, i}^{s, 1}\right|^{2}\right]\right\} \Lambda^{2} \alpha_{1} P_{0}+N_{0}} \\
& \stackrel{(j=1)}{=}\left(\max \left\{\left[\left|h_{0, i}^{s, 1}\right|^{2}\right]\right\} \Lambda^{2} \alpha_{1} P_{0}\right) / N_{0} .
\end{aligned}
$$

The instantaneous bit rate of $U_{i}$ for $i=\{1,2, E\}$ obtained when it decoded the $x_{j}$ symbol for $j=\{2,1\}$ expressed as follows:

$R_{i-x_{j}}^{(\mathrm{MISO})}=\max _{s=[1 \cdots S]}\left\{\left[R_{i-x_{j}}^{(s, 1)}\right]\right\}=\log _{2}\left(1+\max \left\{\left[\gamma_{i-x_{j}}^{(s, 1)}\right]\right\}\right)$.

\subsection{MIMO scenario}

We in this subsection assumed the BS and all users were equipped a multiple antenna like [23, 24]. The number antennae on BS denoted by $S>1$, as in the previous subsection, while the number antennae on $U_{i}$ denoted by
$U>1$. The NOMA network therefore existed $S \times U$ channels from $S$ transmitter antennae at the BS to the $U$ receiver antennae at user $U_{i}$ is expressed as follows:

$$
\mathbf{H}_{\mathbf{0 , i}}^{(\mathbf{M I M O})}=\left[\begin{array}{ccc}
h_{0, i}^{(1,1)} & \cdots & h_{0, i}^{(1, U)} \\
\vdots & \ddots & \vdots \\
h_{0, i}^{(S, 1)} & \cdots & h_{0, i}^{(S, U)}
\end{array}\right] .
$$

Each $s$ th transmitter antenna on BS sends a broadcast beamforming superimposed signal to all $U$ antennae at user $U_{i}$. The received signals at each user $U_{i}$ are expressed as follows:

$$
\begin{aligned}
y_{i}^{(\mathrm{MIMO})}= & \operatorname{matrix}\left[y_{i}^{(s, u)}\right] \\
& s=[1 \cdots S], u=[1 \cdots U] \\
= & \operatorname{matrix}\left[h_{0, i}^{(s, u)}\right]\left(\Lambda \sqrt{\alpha_{1} P_{0}} x_{1}+\Delta \sqrt{\alpha_{2} P_{0}} x_{2}\right)+n_{i}, \\
& s=[1 \cdots S], u=[1 \cdots U]
\end{aligned}
$$

where $h_{0, i}^{(s, u)} \in \mathbf{H}_{\mathbf{0}, \mathbf{i}}^{(\mathbf{M I M O})}$ is the transmission channel from $s$ th transmitter antenna at the BS to the $u$ th receiver antenna at user $U_{i}$ for vector $s=[1 \cdots S]$ and vector $u=[1 \cdots U]$.

The TAS protocol was deployed at the user in this subsection. After selecting the best pairing antenna with one at the BS and one at $U_{i}$, the SINRs therefore obtained at the $U_{i}$ when it detects the $x_{j}$ symbol for $i=\{1,2, E\}$ and $j=\{2,1\}$ are expressed as follows:

$$
\begin{aligned}
\gamma_{i-x_{j}}^{(\mathrm{MIMO})}= & \max \left\{\left[\gamma_{i-x_{j}}^{(s, u)}\right]\right\} \\
& \stackrel{(j=21 \cdots S], u=[1 \cdots U]}{=} \frac{\max \left\{\left[\left|h_{0, i}^{(s, u)}\right|^{2}\right]\right\} \Delta^{2} \alpha_{2} P_{0}}{\max \left\{\left[\left|h_{0, i}^{(s, u)}\right|^{2}\right]\right\} \Lambda^{2} \alpha_{1} P_{0}+N_{0}}
\end{aligned}
$$

$$
\stackrel{(j=1)}{=}\left(\max \left\{\left[\left|h_{0, i}^{(s, u)}\right|^{2}\right]\right\} \Lambda^{2} \alpha_{1} P_{0}\right) / N_{0}
$$

The instantaneous bit rate of $U_{i}$ achieved over MIMO scheme when it decodes the $x_{j}$ symbol is expressed as follows:

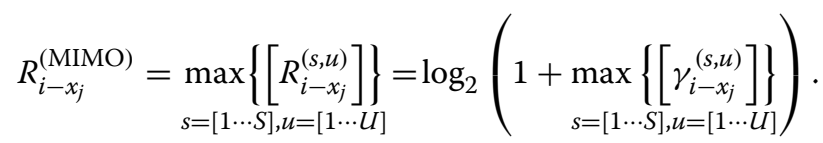




\section{System performance analysis}

In this section, NOP, SOP and system throughput, respectively, is analysed. Note that the users were over Rayleigh fading channels with a respective probability density function (PDF) and cumulative distribution function (CDF) expressed as $f_{\left|h_{0, i}\right|^{2}}=\exp \left(-x / \sigma_{0, i}^{2}\right) / \sigma_{0, i}^{2}$, and $F_{\left|h_{0, i}\right|^{2}}=$ $1-\exp \left(-x / \sigma_{0, i}^{2}\right)$ where $x$ refers to a random independent variable followed by $x \geq 0$, and $\sigma_{0, i}^{2}$ refers to the mean of the channel followed by $\sigma_{0, i}^{2}=E\left[\left|h_{0, i}\right|^{2}\right]$.

\subsection{Non-secrecy outage probability (NOP) without eavesdropper $E$}

Ding et al. [6] investigated a NOMA system with random $\chi$ users. In addition, the authors demonstrated the NOP at $i$ th user for $i \in \chi$ occurred when it cannot successfully decode at least one of data symbols $x_{j}$ for $j=\{\chi, \ldots, i\}$ [14]. Although, the system model (Fig. 1) in this study had only two users. However, the system model can be expanded with a massive $\chi$ users like [6] and [14]. The NOPs at the two users over three individual schemes were respectively presented in terms as follows.

Theorem 1 As shown in Fig. 1, the NOP of signal transmission at $U_{i}$ occurred when $U_{i}$ cannot successfully decode $x_{j}$ symbol for $i=\{1,2\}$ meanwhile $j=\{2, i\}$. Specifically, the NOP at $U_{1}$ and $U_{2}$ will occur when there is one of corresponding the following cases:

- NOP at $U_{1}$ : The NOP of signal transmission at $U_{1}$ will occur when it cannot successfully decode either $x_{2}$ or $x_{1}$ symbol. For clarity, $U_{1}$ over three individual scenarios firstly decoded the $x_{2}$ symbol of $U_{2}$ and obtained SINRs that are given by (2), (6), and (11), respectively. After detecting the $x_{2}$ symbol, the $U_{1}$ removed the $x_{2}$ symbol from the received signal and then $U_{1}$ decoded its own $x_{1}$ symbol and obtained SINRs as shown in (3), (7), and (12). After SIC-ing with assuming the SINRs obtained with implementing perfect, imperfect and fully imperfect SICs, the instantaneous bit rate thresholds then obtained when $U_{1}$ decoded $x_{j}$ symbol for $j=\{2,1\}$, which were given by (4), (8), or (13). Next processing, the instantaneous bit rate thresholds $R_{1-x_{j}}^{(\Psi)}$ for $\Psi=\{$ SISO, MISO, MIMO $\}$ were compared with $U_{j}$ s' bit rate thresholds denoted by $R_{j}^{*}$. The NOP at $U_{1}$ therefore occurred as a result when the instantaneous bit rate threshold $R_{1-x_{j}}^{(\Psi)}$ cannot reach to the bit rate threshold $R_{j}^{*}$ of user $U_{j}$ for $j=\{2,1\}$. In other words, the NOP at $U_{1}$ over three individual scenarios was then expressed as follows:

$$
\Theta_{1}^{(\Psi)}=1-\prod_{j=2}^{1} \Theta_{1-x_{j}}^{(\Psi)}=1-\Theta_{1-x_{2}}^{(\Psi)} \Theta_{1-x_{1}}^{(\Psi)}
$$

$$
=1-\left\{\operatorname{Pr}\left\{R_{i-x_{2}}^{(\Psi)} \geq R_{2}^{*}\right\} \text { and } \operatorname{Pr}\left\{R_{i-x_{1}}^{(\Psi)} \geq R_{1}^{*}\right\}\right\} \text {. }
$$

It is worth to noting that the upper limit of Eq. (14) with $j=2$ indicates the number of current $\chi$ users have joined the network. By replacing the upper limit small value $j$ with a massive $\chi$, Eq. (14) can be used to investigate NOP at the strongest user over NOMA network with a massive $\chi$ user scenario like [6] or [14]. However, the aim of this study was to examined the impact of antennae on system performance, which is presented in the following sections. We were therefore limited to only $\chi=2$ users without losing NOMA key features.

- NOP at $U_{2}$ : The NOP of signal transmission at $U_{2}$ will occur when it cannot successfully decode its own $x_{2}$ symbol by treating $x_{1}$ symbol as interference, assuming that the SINRs obtained with perfect, imperfect and fully imperfect SIC, respectively, over three individual scenario as shown (2) for the SISO scenario, (6) for the MISO scenario and (11) for the MIMO scenario, where $i=j=2$. After SIC-ing, the instantaneous bit rate threshold is obtained when $U_{2}$ decodes its own symbol $x_{2}$, which is similarly given by (4), (8), and (13), where $i=j=2$. Further processing, the instantaneous bit rate thresholds $R_{2-x_{2}}^{(\Psi)}$ for $\Psi=\{$ SISO, MISO, MIMO $\}$ were compared with its own bit rate threshold $R_{2}^{*}$. The NOP at $U_{2}$ therefore occurred as a result when $R_{2-x_{2}}^{(\Psi)}$ cannot reach to the bit rate threshold $R_{2}^{*}$. In other words, the NOP at $U_{2}$ over three individual scenarios is expressed as follows:

$$
\begin{aligned}
\Theta_{2}^{(\Psi)} & =1-\Theta_{2-x_{2}}^{(\Psi)}=\operatorname{Pr}\left\{R_{2-x_{2}}^{(\Psi)}<R_{2}^{*}\right\} \\
& =1-\operatorname{Pr}\left\{R_{2-x_{2}}^{(\Psi)} \geq R_{2}^{*}\right\} .
\end{aligned}
$$

\subsubsection{NOP at users over SISO scheme}

Remarks 1 Through NOP conditions as shown in (14) and (15) in Theorem 1, this study obtained the NOP at users over SISO scheme.

First, the NOP at $U_{1}$ over SISO scenario is obtained and expressed in closed form as follows:

$\Theta_{1}^{(\mathrm{SISO})}=1-\left\{\operatorname{Pr}\left\{R_{1-x_{2}}^{(\mathrm{SISO})} \geq R_{2}^{*}\right\}\right.$ and $\left.\operatorname{Pr}\left\{R_{1-x_{1}}^{(\mathrm{SISO})} \geq R_{1}^{*}\right\}\right\}$

$$
=1-e^{-\left(\frac{\gamma_{2}^{*}-1}{\left(\Delta^{2} \alpha_{2}-\Lambda^{2} \alpha_{1}\left(\gamma_{2}^{*}-1\right)\right) \rho_{0} \sigma_{0,1}^{2}}+\frac{\gamma_{1}^{*}-1}{\Lambda^{2} \alpha_{1} \rho_{0} \sigma_{0,1}^{2}}\right)},
$$

where $\gamma_{j}^{*}=2^{R_{j}^{*}}$ for $j=\{2,1\}$.

Through observation (17), it was simple to note that when the SIC was perfect, $U_{1}$ certainly detected the $x_{1}$ symbol 
but could not successfully detect its own $x_{1}$ symbol. $U_{1}$ therefore obtained the worst QoS. This issue was verified by analysis and simulation results shown in the next section.

Second, the NOP at $U_{2}$ over SISO scenario is easily obtained and expressed in closed form as follows:

$$
\begin{aligned}
\Theta_{2}^{(\mathrm{SISO})} & =1-R_{1-x_{2}}^{(\mathrm{SISO})} \geq R_{2}^{*} \\
& =1-e^{-\frac{\gamma_{2}^{*}-1}{\left(\Delta^{2} \alpha_{2}-\Lambda^{2} \alpha_{1}\left(\gamma_{2}^{*}-1\right)\right) \rho_{0} \sigma_{0,2}^{2}}},
\end{aligned}
$$

where $\Lambda^{2}=0$ for perfect SIC, $0<\Lambda^{2}<1$ for imperfect SIC and $\Lambda^{2}=1$ for fully imperfect SIC. By observation (19), it was simple to note that when the SIC was perfect, $U_{2}$ obtained the best QoS.

See Appendix for proof.

\subsubsection{NOP at users over MISO scheme}

Remarks 2 The MISO scheme in this study assumed that the BS was equipped with a multiple transmitter antenna while the users were still equipped with a single receiver antenna, such as [35]. It was important to remember that $S$ denoted the number of transmitter antennae at the BS, where $S>1$. Therefore, The matrix channels from the $B S$ to the user $U_{i}$ are $\mathbf{H}_{0, i}^{(\mathrm{MISO})}=$ $\left[h_{0, i}^{(1,1)} \cdots h_{0, i}^{(s, 1)} \cdots h_{0, i}^{(S, 1)}\right]$ for $s \in S$. TAS protocol was deployed.

The NOP conditions at $U_{1}$ over MISO scenario are given as follows:

$$
\begin{aligned}
\Theta_{1}^{(\mathrm{MISO})}=1 & -\operatorname{Pr}\left\{\max _{s=[1 \cdots S]}\left\{\left[R_{1-x_{2}}^{(s, 1)}\right]\right\} \geq R_{2}^{*}\right\} \\
& \times \operatorname{Pr}\left\{\max _{s=[1 \cdots S]}\left\{\left[R_{1-x_{1}}^{(s, 1)}\right]\right\} \geq R_{1}^{*}\right\} .
\end{aligned}
$$

Equation (20) is obtained in closed form as follows:

$$
\begin{aligned}
\Theta_{1}^{(M I S O)}= & \prod_{s=1}^{S}\left(1-e^{-\frac{\gamma_{2}^{*}-1}{\left(\Delta^{2} \alpha_{2}-\Lambda^{2} \alpha_{1}\left(\gamma_{2}^{*}-1\right)\right) \rho_{0} \sigma_{0,1}^{2}}}\right) \\
& +\left(1-\prod_{s=1}^{S}\left(1-e^{-\frac{\gamma_{2}^{*}-1}{\left(\Delta^{2} \alpha_{2}-\Lambda^{2} \alpha_{1}\left(\gamma_{2}^{*}-1\right)\right) \rho_{0} \sigma_{0,1}^{2}}}\right)\right) \\
& \times \prod_{s=1}^{S}\left(1-e^{-\frac{\gamma_{1}^{*}-1}{\Lambda^{2} \alpha_{1} \rho_{0} \sigma_{0,1}^{2}}}\right) .
\end{aligned}
$$

In addition, the approximation in closed form of NOP at $U_{1}$ is obtained by using the PDF as shown in (57) and (58) for $0 \leq x<\frac{\Delta^{2} \alpha_{2}}{\Lambda^{2} \alpha_{1}}$ and expressed as follows:

$$
\begin{aligned}
\Theta_{1}^{(\mathrm{MISO})}=1 & -\left(1-\sum_{s=0}^{S} \frac{(-1)^{s} S !}{s !(S-s) !} e^{-\frac{s\left(\gamma_{2}^{*}-1\right)}{\left(\Delta^{2} \alpha_{2}-\Lambda^{2} \alpha_{1}\left(\gamma_{2}^{*}-1\right)\right) \rho_{0} \sigma_{0,1}^{2}}}\right) \\
& \times\left(1-\sum_{s=0}^{S} \frac{(-1)^{s} S !}{s !(S-s) !} e^{-\frac{s\left(\gamma_{1}^{*}-1\right)}{\Lambda^{2} \alpha_{1} \rho_{0} \sigma_{0,1}^{2}}}\right) .
\end{aligned}
$$

However, the NOP conditions at the $U_{2}$ over MISO scenario are given as follows:

$\Theta_{2}^{(\mathrm{MISO})}=1-\Theta_{2-x_{2}}^{(\mathrm{MISO})}=1-\operatorname{Pr}\left\{\max _{s=[1 \cdots S]}\left\{\left[R_{2-x_{2}}^{(s, 1)}\right]\right\} \geq R_{2}^{*}\right\}$.

Equation (23) is obtained in closed form as follows:

$$
\Theta_{2}^{(\mathrm{MISO})}=\prod_{s=1}^{S}\left(1-e^{-\frac{\gamma_{2}^{*}-1}{\left(\Delta^{2} \alpha_{2}-\Lambda^{2} \alpha_{1}\left(\gamma_{2}^{*}-1\right)\right) \rho_{0} \sigma_{0,2}^{2}}}\right) .
$$

Similarly, the approximation in closed form of NOP at $\mathrm{U}_{2}$ over MISO scenario is obtained by using the PDF as shown (57) for $0 \leq x<\frac{\Delta^{2} \alpha_{2}}{\Lambda^{2} \alpha_{1}}$ and expressed as follows:

$$
\Theta_{2}^{(\mathrm{MISO})}=\sum_{s=0}^{S} \frac{(-1)^{s} S !}{s !(S-s) !} e^{-\frac{s\left(\gamma_{1}^{*}-1\right)}{\left(\Delta^{2} \alpha_{2}-\Lambda^{2} \alpha_{1}\left(\gamma_{2}^{*}-1\right)\right) \rho_{0} \sigma_{0,1}^{2}}} .
$$

See Appendix for the proof.

\subsubsection{NOP at users over MIMO scheme}

Remarks 3 In this section, this study assumed that the $B S$ and all users are equipped with a multiple antenna. It is important to remember that $U$ denotes the number of receiver antennae at the users, where $U>1$, while $S$ denotes the number of transmitter antennae at the BS, where $S>1$. Therefore, the matrix channels from the sth transmitter antenna at the $B S$ to the uth receiver antenna at the ith user for vectors $s=[1 \cdots S]$, and $u=[1 \cdots U]$ are $\mathbf{H}_{\mathbf{0}, \mathbf{i}}^{(\mathbf{M I M O})}$. The TAS protocol was deployed in this scenario.

The NOP conditions at $U_{1}$ over MIMO scenario are given as follows:

$$
\Theta_{1}^{(\mathrm{MISO})}=1-\prod_{j=2}^{1} \Theta_{1-x_{j}}^{(\mathrm{MISO})}=1-\Theta_{1-x_{2}}^{(\mathrm{MISO})} \Theta_{1-x_{1}}^{(\mathrm{MISO})}
$$




$$
\begin{gathered}
=1-\left\{\operatorname{Pr}\left\{\begin{array}{l}
\left.\max \left\{\left[R_{1-x_{2}}^{(s, u)}\right]\right\} \geq R_{2}^{*}\right\}, \\
s=[1 \cdots S], u=[1 \cdots U]
\end{array}\right\}\right. \\
\operatorname{Pr}\left\{\begin{array}{l}
\left.\max \left\{\left[R_{1-x_{1}}^{(s, u)}\right]\right\} \geq R_{1}^{*}\right\} \\
s=[1 \cdots S], u=[1 \cdots U]
\end{array}\right\} .
\end{gathered}
$$

Equation (26) is obtained in closed form as follows:

$$
\begin{aligned}
\Theta_{1}^{(\mathrm{MISO})}= & \prod_{u=1}^{U} \prod_{s=1}^{S}\left(1-e^{-\frac{\gamma_{2}^{*}-1}{\left(\Delta^{2} \alpha_{2}-\Lambda^{2} \alpha_{1}\left(\gamma_{2}^{*}-1\right)\right) \rho_{0} \sigma_{0,1}^{2}}}\right) \\
+ & \left(1-\prod_{u=1}^{U} \prod_{s=1}^{S}\left(1-e^{-\frac{\gamma_{2}^{*}-1}{\left(\Delta^{2} \alpha_{2}-\Lambda^{2} \alpha_{1}\left(\gamma_{2}^{*}-1\right)\right) \rho_{0} \sigma_{0,1}^{2}}}\right)\right) \\
& \times \prod_{u=1}^{U} \prod_{s=1}^{S}\left(1-e^{-\frac{\gamma_{1}^{*}-1}{\Lambda^{2} \alpha_{1} \rho_{0} \sigma_{0,1}^{2}}}\right)
\end{aligned}
$$

In addition, the approximation in closed form of NOP at $U_{1}$ over MIMO scenario can be also obtained by using the PDF as shown (55) and expressed as follows:

$$
\begin{aligned}
\Theta_{1}^{(\mathrm{MISO})}=1 & -\left(1-\sum_{n=0}^{N=S U} \frac{(-1)^{s} N !}{n !(N-n) !} e^{-\frac{n\left(\gamma_{2}^{*}-1\right)}{\left(\Delta^{2} \alpha_{2}-\Lambda^{2} \alpha_{1}\left(\gamma_{2}^{*}-1\right)\right) \rho_{0} \sigma_{0,1}^{2}}}\right) \\
& \times\left(1-\sum_{n=0}^{N=S U} \frac{(-1)^{s} N !}{n !(N-n) !} e^{-\frac{n\left(\gamma_{1}^{*}-1\right)}{\Lambda^{2} \alpha_{1} \rho_{0} \sigma_{0,1}^{2}}}\right) .
\end{aligned}
$$

However, the NOP conditions at $U_{2}$ over MIMO scenario are given as follows:

$$
\begin{aligned}
\Theta_{2}^{(\mathrm{MISO})} & =1-\Theta_{2-x_{2}}^{(\mathrm{MISO})} \\
& =1-\operatorname{Pr}\left\{\begin{array}{l}
\left.\max \left\{\left[R_{2-x_{2}}^{(s, u)}\right]\right\} \geq R_{2}^{*}\right\} . \\
s=[1 \cdots S], u=[1 \cdots U]
\end{array}\right\} . .
\end{aligned}
$$

Equation (29) is obtained in closed form as follows:

$$
\Theta_{2}^{(\mathrm{MISO})}=\prod_{u=1}^{U} \prod_{s=1}^{S}\left(1-e^{-\frac{\gamma_{2}^{*}-1}{\left(\Delta^{2} \alpha_{2}-\Lambda^{2} \alpha_{1}\left(\gamma_{2}^{*}-1\right)\right) \rho_{0} \sigma_{0,1}^{2}}}\right) .
$$

Similarly, the approximation in closed form of NOP at $\mathrm{U}_{2}$ over MIMO scenario is also obtained by using the PDF (55) and expressed as follows:

$$
\Theta_{2}^{(\mathrm{MISO})}=\sum_{n=0}^{N=S U} \frac{(-1)^{n} N !}{n !(N-n) !} e^{-\frac{n\left(\gamma_{1}^{*}-1\right)}{\left(\Delta^{2} \alpha_{2}-\Lambda^{2} \alpha_{1}\left(\gamma_{2}^{*}-1\right)\right) \rho_{0} \sigma_{0,1}^{2}}}
$$

See Appendix for the proof.

\subsection{Secrecy outage probability (SOP) with eavesdropper $E$} In this investigation, this study assumed an eavesdropper $E$ existed in the NOMA network. Eavesdropper $E$ over three individual scenarios received signals by substituting its own channel $h_{0, E}^{(\Psi)}$ into (1), (5) or (10). The eavesdropper $E$ can also detect $x_{1}$ or $x_{2}$ data symbol of $U_{1}$ or $U_{2}$ when it eavesdrops $U_{1}$ or $U_{2}$. For clarity, the SINRs are obtained at the eavesdropper $E$ when it decodes the $x_{2}$ symbol by substituting $\left|h_{0, E}^{(\Psi)}\right|^{2}$ into (2), (6) or (11). However, the eavesdropper $E$ can also detect and remove $x_{2}$ symbol from received signal and then detect the $x_{1}$ symbol when it eavesdropped $U_{1}$. The instantaneous bit rate threshold of the eavesdropper $E$ over three individual scenarios is therefore obtained from (4), (8) or (13).

The secure instantaneous bit rate of $U_{i}$ for $i=\{1,2\}$ over SISO, MISO and MIMO schemes, respectively, are expressed as follows:

$$
\tilde{R}_{i-x_{j}}^{(\mathrm{SISO})}=\max \left\{R_{i-x_{j}}^{(\mathrm{SISO})}-R_{E-x_{j}}^{(\mathrm{SISO})}, 0\right\},
$$

$$
\begin{aligned}
\tilde{R}_{i-x_{j}}^{(\mathrm{MISO})} & =\max \left\{R_{i-x_{j}}^{(\mathrm{MISO})}-R_{E-x_{j}}^{(\mathrm{MISO})}, 0\right\} \\
& =\max \left\{\max _{s=[1 \cdots S]}\left\{\left[R_{i-x_{j}}^{(s, 1)}\right]\right\}-\max _{s=[1 \cdots S]}\left\{\left[R_{E-x_{j}}^{(s, 1)}\right]\right\}, 0\right\},
\end{aligned}
$$

and

$$
\begin{aligned}
& \tilde{R}_{i-x_{j}}^{(\mathrm{MIS})}=\max \left\{R_{i-x_{j}}^{(\mathrm{MISO})}-R_{E-x_{j}}^{(\mathrm{MISO})}, 0\right\}
\end{aligned}
$$

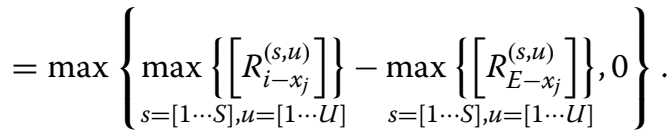

Theorem 1 SOP at the $U_{i}$ over three individual scenarios is the probability that the secure instantaneous bit rate given by (30), (32) or (34) cannot reach the $U_{i} s^{\prime}$ bit rate threshold $R_{i}^{*}$. In other words, the SOP at $U_{i}$ for $i=\{1,2\}$ can be respectively expressed as follows:

$$
\tilde{\Theta}_{1}^{(\Psi)}=\operatorname{Pr}\left\{\tilde{R}_{1-x_{2}}^{(\Psi)}<R_{2}^{*}\right\}+\operatorname{Pr}\left\{\tilde{R}_{1-x_{2}}^{(\Psi)} \geq R_{2}^{*}, \tilde{R}_{1-x_{1}}^{(\Psi)}<R_{1}^{*}\right\},
$$

and

$$
\tilde{\Theta}_{2}^{(\Psi)}=\operatorname{Pr}\left\{\tilde{R}_{2-x_{2}}^{(\Psi)}<R_{2}^{*}\right\}=1-\operatorname{Pr}\left\{\tilde{R}_{2-x_{2}}^{(\Psi)} \geq R_{2}^{*}\right\}
$$

where $\Psi=\{\mathrm{SISO}$, MISO, MIMO $\}$.

\subsubsection{SOP at users over SISO scheme}

Remarks 4 In this subsection, this study investigated the SOP at legitimate user $U_{i}$ for $i=\{1,2\}$ over SISO scheme 
as the system model in [42]. As with (33) in Theorem 2, the $S O P$ at $U_{1}$ over SISO scheme can be rewritten, solved and expressed in closed form as follows:

$$
\begin{aligned}
\tilde{\Theta}_{1}^{(\mathrm{SISO})}= & \operatorname{Pr}\left\{\max \left\{R_{1-x_{2}}^{(\mathrm{SISO})}-R_{E-x_{2}}^{(\mathrm{SISO})}, 0\right\}<R_{2}^{*}\right\} \\
+ & \operatorname{Pr}\left\{\max \left\{R_{1-x_{2}}^{(\mathrm{SISO})}-R_{E-x_{2}}^{(\mathrm{SISO})}, 0\right\} \geq R_{2}^{*},\right. \\
& \left.\max \left\{R_{1-x_{1}}^{(\mathrm{SISO})}-R_{E-x_{1}}^{(\mathrm{SISO})}, 0\right\}<R_{1}^{*}\right\} \\
= & 1-\frac{\Delta^{2} \alpha_{2} \varpi \pi}{2 W \rho_{0} \sigma_{0, E}^{2}} \sum_{w=1}^{W} \frac{\sqrt{1-\lambda^{2}}}{\left(\Delta^{2} \alpha_{2}-\Lambda^{2} \alpha_{1} \beta\right)^{2}} e^{-\theta_{1}} \\
\times & \frac{\sigma_{0,1}^{2}}{\gamma_{1}^{*} \sigma_{0, E}^{2}+\sigma_{0,1}^{2}} e^{-\frac{\gamma_{1}^{*}-1}{\Lambda^{2} \alpha_{1} \rho_{0} \sigma_{0,1}^{2}}},
\end{aligned}
$$

where $\theta_{1}=\frac{\gamma_{2}^{*}(1+\beta)-1}{\left(\Delta^{2} \alpha_{2}-\Lambda^{2} \alpha_{1}\left(\gamma_{2}^{*}(1+\beta)-1\right)\right) \rho_{0} \sigma_{0,1}^{2}}+$ $\frac{\beta}{\left(\Delta^{2} \alpha_{2}-\Lambda^{2} \alpha_{1} \beta\right) \rho_{0} \sigma_{0, E}^{2}}, \beta=\frac{\varpi(\lambda+1)}{2}, \lambda=\cos \left(\frac{2 w-1}{2 W} \pi\right)$, and $\varpi=\frac{1}{\Lambda^{2}\left(1-\alpha_{1}\right) \gamma_{1}^{*}}-1+\Xi<\frac{\Delta^{2} \alpha_{2}}{\Lambda^{2} \alpha_{1}}$, while $\Xi$ is the approximate coefficient with $0<\Xi<\frac{\Delta^{2} \alpha_{2}}{\Lambda^{2} \alpha_{1}}-\left(\frac{1}{\Lambda^{2}\left(1-\alpha_{1}\right) \gamma_{1}^{*}}-1\right)$ for the case of imperfect SIC, otherwise $\Xi=0$. It is important to note that $W$ referred the accuracy coefficient. Meanwhile the coefficient $W$ is an increasingly large value, the SOP analysis results at $U_{1}$ become increasingly more accurate.

However, the SOP at $U_{2}$ over SISO scheme is also rewritten, solved and expressed in closed form as follows:

$$
\begin{aligned}
\tilde{\Theta}_{2}^{(\mathrm{SISO})} & =\operatorname{Pr}\left\{\max \left\{R_{2-x_{2}}^{(\mathrm{SISO})}-R_{E-x_{2}}^{(\mathrm{SISO})}, 0\right\}<R_{2}^{*}\right\} \\
& =1-\frac{\Delta^{2} \alpha_{2} \varpi \pi}{2 \mathrm{~W} \rho_{0} \sigma_{0, E}^{2}} \sum_{w=1}^{W} \frac{\sqrt{1-\lambda^{2}}}{\left(\Delta^{2} \alpha_{2}-\Lambda^{2} \alpha_{1} \beta\right)^{2}} e^{-\theta_{2}}
\end{aligned}
$$

$$
\begin{aligned}
& \text { where } \theta_{2}=\frac{\gamma_{2}^{*}(1+\beta)-1}{\frac{\beta}{\left(\Delta^{2} \alpha_{2}-\Lambda^{2} \alpha_{1}\left(\gamma_{2}^{*}(1+\beta)-1\right) \rho_{0} \sigma_{0,2}^{2}\right.}}+ \\
& \frac{\left(\Delta^{2} \alpha_{2}-\Lambda^{2} \alpha_{1} \beta\right) \rho_{0} \sigma_{0, E}^{2}}{\text { See Appendix for the proof. }}
\end{aligned}
$$

\subsubsection{SOP at user over MISO scheme}

Remarks 5 In this subsection, this study investigated the SOP at user $U_{i}$ for $i=\{1,2\}$ over MISO scheme. As with (35) in Theorem 2, the SOP at $U_{1}$ over MISO scheme can be rewritten and expressed as follows:

$$
\begin{aligned}
& \tilde{\Theta}_{1}^{(\mathrm{MISO})}=\operatorname{Pr}\left\{\max \left\{\max \left\{\left[R_{1-x_{2}}^{(s, 1)}\right]\right\}-\max \left\{\left[R_{E-x_{2}}^{(s, 1)}\right]\right\}, 0\right\}<R_{2}^{*}\right\}
\end{aligned}
$$

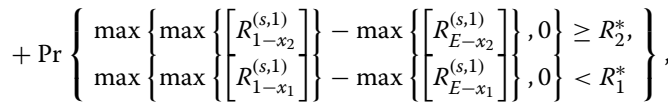

where vector $s=[1 \cdots S]$.

The CDF of $\gamma_{i-x_{j}}^{(\mathrm{MISO})}$ for $i=\{1,2, E\}$ are respectively given by (57) for $j=2$ or (58) for $j=1$.

The PDF of $\gamma_{i-x_{j}}^{(\mathrm{MISO})}$ for $i=\{1,2, E\}$ and $j=\{2,1\}$ is respectively given by (59) or (60).

Through Eq. (41), the SOP at $U_{1}$ can be obtained and expressed in closed form as follows:

$$
\begin{aligned}
& \tilde{\Theta}_{1}^{(M I S O)} \\
& =\sum_{s=0}^{S} \frac{(-1)^{s} S ! \sigma_{0,1}^{2}}{s !(S-s) !\left(s \gamma_{1}^{*} \sigma_{0, E}^{2}+\sigma_{0,1}^{2}\right)} e^{-\frac{s\left(\gamma_{1}^{*}-1\right)}{\Lambda^{2} \alpha_{1} \rho_{0} \sigma_{0,1}^{2}}} \\
& \quad \times\left(G_{1}+e^{\left.-\frac{\omega}{\left(\Delta^{2} \alpha_{2}-\Lambda^{2} \alpha_{1} \sigma\right) \rho_{0} \sigma_{0, E}^{2}}\right)}\right. \\
& =\sum_{s=0}^{S} \frac{(-1)^{s} S ! \sigma_{0,1}^{2}}{s !(S-s) !\left(s \gamma_{1}^{*} \sigma_{0, E}^{2}+\sigma_{0,1}^{2}\right)} e^{-\frac{s\left(\gamma_{1}^{*}-1\right)}{\Lambda^{2} \alpha_{1} \rho_{0} \sigma_{0,1}^{2}}} \\
& \quad \times \frac{\varpi \pi \Delta^{2} \alpha_{2} S !}{2 \rho_{0} W \sigma_{0, E}^{2}} \sum_{s=0}^{S} \sum_{w=1}^{W} \frac{(-1)^{s} \sqrt{1-\lambda^{2}} e^{-\Phi_{1}}}{s !(S-s) !\left(\Delta^{2} \alpha_{2}-\Lambda^{2} \alpha_{1} \beta\right)^{2}} \\
& \quad+e^{-\frac{\omega}{\left(\Delta^{2} \alpha_{2}-\Lambda^{2} \alpha_{1} \sigma\right) \rho_{0} \sigma_{0, E}^{2}}},
\end{aligned}
$$

where $G_{1}$ was given by (70).

The SOP at $U_{2}$ as with (36) in Theorem 2 can be obtained and expressed in closed form as follows:

$\tilde{\Theta}_{2}^{(\mathrm{MISO})}$

$$
\begin{aligned}
& =1-\operatorname{Pr}\left\{\max \left\{\max _{s=[1 \cdots S]}\left\{\left[R_{2-x_{2}}^{(s, 1)}\right]\right\}-\max _{s=[1 \cdots S]}\left\{\left[R_{E-x_{2}}^{(s, 1)}\right]\right\}, 0\right\} \geq R_{2}^{*}\right\} \\
& =G_{2}+e^{-\frac{\sigma}{\left(\Delta^{2} \alpha_{2}-\Lambda^{2} \alpha_{1} \sigma\right) \rho_{0} \sigma_{0, E}^{2}}}
\end{aligned}
$$

$$
\begin{aligned}
= & \frac{\varpi \pi \Delta^{2} \alpha_{2} S !}{2 W \rho_{0} \sigma_{0, E}^{2}} \sum_{s=0}^{S} \sum_{w=1}^{W} \frac{(-1)^{s} \sqrt{1-\lambda^{2}} e^{-\Phi_{2}}}{s !(S-s) !\left(\Delta^{2} \alpha_{2}-\Lambda^{2} \alpha_{1} \beta\right)^{2}} \\
& +e^{-\frac{\omega}{\left(\Delta^{2} \alpha_{2}-\Lambda^{2} \alpha_{1} \varpi\right) \rho_{0} \sigma_{0, E}^{2}}},
\end{aligned}
$$

where $\Phi_{i}=\frac{s\left(\gamma_{2}^{*}(1+\beta)-1\right)}{\left(\Delta^{2} \alpha_{2}-\Lambda^{2} \alpha_{1}\left(\gamma_{2}^{*}(1+\beta)-1\right)\right) \rho_{0} \sigma_{0, i}^{2}}+$ $\frac{\beta}{\left(\Delta^{2} \alpha_{2}-\Lambda^{2} \alpha_{1} \beta\right) \rho_{0} \sigma_{0, E}^{2}}$ for $i=\{1,2\}$, and $G_{2}$ is given by $(70)$ by substituting $i=2$.

See Appendix for the proof.

\subsubsection{SOP at user over MIMO scheme}

Remarks 6 In this subsection, this study investigated the SOP at $U_{i}$ for $i=\{1,2\}$ over MIMO scenario. The users were also equipped a multiple receiver antenna. As with (35) in Theorem 2, the SOP of $U_{1}$ therefore can therefore be 
obtained a nd expressed in closed form as follows:

$$
\begin{aligned}
& \tilde{\Theta}_{1}^{(\mathrm{MISO})}=1-\operatorname{Pr}\left\{\max \left\{\begin{array}{l}
\max \left\{\left[R_{1-x_{1}}^{(s, u)}\right]\right\}-\max \left\{\left[R_{E-x_{1}}^{(s, u)}\right]\right\}, 0 \\
s=[1 \cdots S], u=[1 \cdots U]
\end{array}\right\} \geq R_{1}^{*}\right\}
\end{aligned}
$$

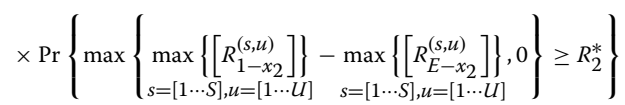

$$
\begin{aligned}
& =\sum_{n=0}^{N=S U} \frac{(-1)^{n}(N) ! \sigma_{0,1}^{2}}{n !(N-n) !\left(n \gamma_{1}^{*} \sigma_{0, E}^{2}+\sigma_{0,1}^{2}\right)} e^{-\frac{n\left(\gamma_{1}^{*}-1\right)}{\Lambda^{2} \alpha_{1} \rho_{0} \sigma_{0,1}^{2}}} \\
& \times \frac{\varpi \pi \Delta^{2} \alpha_{2} S !}{2 \rho_{0} W \sigma_{0, E}^{2}} \sum_{s=0}^{S} \sum_{w=1}^{W} \frac{(-1)^{s} \sqrt{1-\lambda^{2}} e^{-\Phi_{1}}}{s !(S-s) !\left(\Delta^{2} \alpha_{2}-\Lambda^{2} \alpha_{1} \beta\right)^{2}} \\
& +e^{-\frac{\varpi}{\left(\Delta^{2} \alpha_{2}-\Lambda^{2} \alpha_{1} \varpi\right) \rho_{0} \sigma_{0, E}^{2}}} \text {. }
\end{aligned}
$$

However, the SOP at $U_{2}$ over MIMO scenario can be obtained and expressed in closed form as follows:

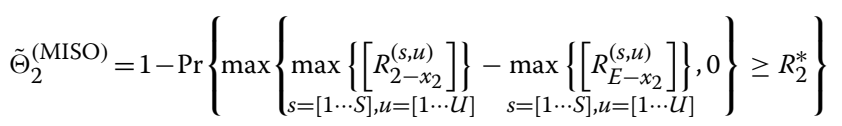

$$
\begin{aligned}
= & \frac{\varpi \pi \Delta^{2} \alpha_{2} N !}{2 W \rho_{0} \sigma_{0, E}^{2}} \sum_{n=0}^{N} \sum_{w=1}^{W} \frac{(-1)^{n} \sqrt{1-\lambda^{2}} e^{-\Phi_{2}}}{n !(N-n) !\left(\Delta^{2} \alpha_{2}-\Lambda^{2} \alpha_{1} \beta\right)^{2}} \\
& +e^{-\frac{\omega}{\left(\Delta^{2} \alpha_{2}-\Lambda^{2} \alpha_{1} \varpi\right) \rho_{0} \sigma_{0, E}^{2}}} .
\end{aligned}
$$

\section{See Appendix for the proof.}

\subsection{System throughput}

The system throughput is the sum of achievable received bit rate at all $U_{i}$ for $i=\{1,2\}$ which was denoted by $P_{\text {sys }}^{(\Psi)}$ [47]. The system throughput can therefore be computed and expressed as follows:

$$
P_{\text {sys }}^{(\Psi)}=P_{1}^{(\Psi)}+P_{2}^{(\Psi)}=\left(1-\Theta_{1}^{(\Psi)}\right) R_{1}^{*}+\left(1-\Theta_{2}^{(\Psi)}\right) R_{2}^{*},
$$

where $\Psi=$ \{SISO, MISO, MIMO $\}$.

\section{Numerical results and discussions}

In this section, this study presents the analysis results and Monte Carlo simulation results obtained from the investigation in the previous sections. Due to Kong et al. [40] confirmed that the factors include number of users, path-loss exponent, the number of antennae impacted on system performance. We therefore set the parameters for two users $U_{1}$ and $U_{2}$ and an eavesdropper $E$, path-loss exponent $r=4$ [41]. We subjected the parameters to analysis and simulate as shown in Table 1.
Note that in all figures, the markers indicate the analysis results while the solid or dashed lines indicate the Monte Carlo simulation results. The simulation results were based on the investigation of $10^{6}$ random samples. Monte Carlo simulation results were used to compare and verify the analysis results.

\subsection{Results and discussions for perfect/imperfect/fully imperfect sIC}

In this subsection, this study investigated the NOP and SOP performance at the users over SISO scheme. The PA coefficients were also given by two PA strategies by [6] with $\left(\alpha_{1}^{[6]}=0.33333, \alpha_{2}^{[6]}=0.66667\right)$, and [14] with $\left(\alpha_{1}^{[14]}=0.45136, \alpha_{2}^{[14]}=0.54864\right)$. Figure 2a plots the NOP results at $U_{1}$ with implementing perfect $\left(\Lambda^{2}=0\right)$, imperfect $\left(\Lambda^{2}=0.5\right)$ and fully imperfect $\left(\Lambda^{2}=1\right)$ SICs, respectively. It is interesting to observe the results obtained through Fig. 2a. Although $U_{1}$ implemented perfect SIC, the NOP performance at $U_{1}$, however, obtained the worst results. For clarity, $U_{1}$ had two SIC phases. The first SIC phase decoded the $x_{2}$ symbol and then removing $x_{2}$ symbol from the received signal. For the perfect SIC case with coefficient $\Lambda^{2}=0, U_{1}$ therefore easily decoded the $x_{2}$ symbol with only interference $n_{1}$. In the second SIC phase, $U_{1}$ cannot successfully decode its own $x_{1}$ symbol due to $\operatorname{Pr}\left\{R_{1-x_{1}}^{(\mathrm{SISO})} \geq R_{1}^{*}\right\}=0$. The NOP results at $U_{1}$ obtained through PA strategy given by [14] better than another strategy given by [6] at the SNRs $\rho_{0} \rightarrow \infty$.

Figure $2 \mathrm{~b}$ also plots the SOP results at $U_{1}$ and also implemented with perfect/imperfect/fully imperfect SIC at $U_{1}$. The SOP results at $U_{1}$ assuming perfect $\operatorname{SIC}\left(\Lambda^{2}=\right.$ $0)$ still obtained the worst results at low SNRs $\rho_{0}<40$ $\mathrm{dB}$. The SOP results at $U_{1}$ assuming fully imperfect SIC $\left(\Lambda^{2}=1\right)$ still obtained the best results at SNRs $\rho_{0}<50$ $\mathrm{dB}$. However, the SOP results at $U_{1}$ assuming imperfect SIC $\left(\Lambda^{2}=0.5\right)$ obtained the best results when SNRs $\rho_{0}$

Table 1 Simulation parameters

\begin{tabular}{lll}
\hline Symbols & Values & Description \\
\hline$\sigma_{0,1}^{2}=E\left[\left|h_{0,1}\right|^{2}\right]$ & 0.0625 for $(\mathrm{d}=2, \mathrm{r}=4)$ & Channel gain from BS to $U_{1}$ \\
$\sigma_{0,2}^{2}=E\left[\left|h_{0,2}\right|^{2}\right]$ & 0.0514 for $(\mathrm{d}=2.1, \mathrm{r}=4)$ & Channel gain from BS to $U_{2}$ \\
$\sigma_{0, E}^{2}=E\left[\left|h_{0, E}\right|^{2}\right]$ & 0.0514 for $(\mathrm{d}=2.1, \mathrm{r}=4)$ & Channel gain from BS to $E$ \\
$\alpha_{1}=\sigma_{0,2}^{2} / \delta$ & 0.4514 for $\delta=\sum_{i=1}^{2} \sigma_{0, i}^{2}$ & Allocation power factor of $U_{1}$ \\
$\alpha_{2}=\sigma_{0,1}^{2} / \delta$ & 0.5486 for $\delta=\sum_{i=1}^{2} \sigma_{0, i}^{2}$ & Allocation power factor of $U_{2}$ \\
$R_{1}^{*}$ & $0.1 \mathrm{BPCU}$ & Bit rate threshold of $U_{1}$ \\
$R_{2}^{*}$ & $0.1 \mathrm{BPCU}$ & Bit rate threshold of $U_{2}$ \\
$\rho_{0}$ & Optional & SNRs at BS \\
$S$ & Optional & The antennae at BS \\
$U$ & Optional & The antennae $U_{i}$ and $E$ \\
\hline
\end{tabular}

Note: Monte Carlo simulation based on $10^{6}$ random samples of each $h_{0, i}$ channel 


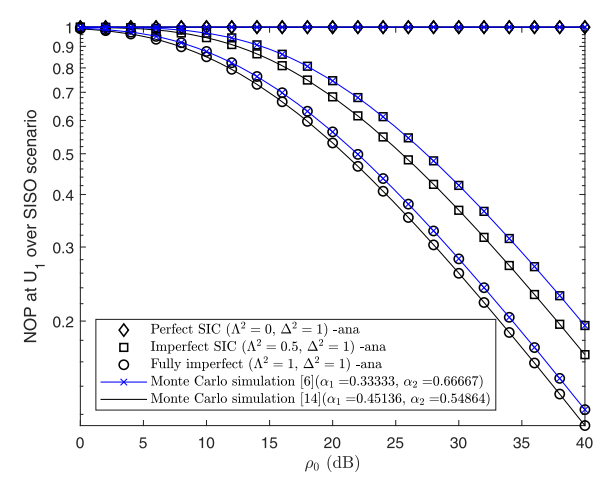

(a)

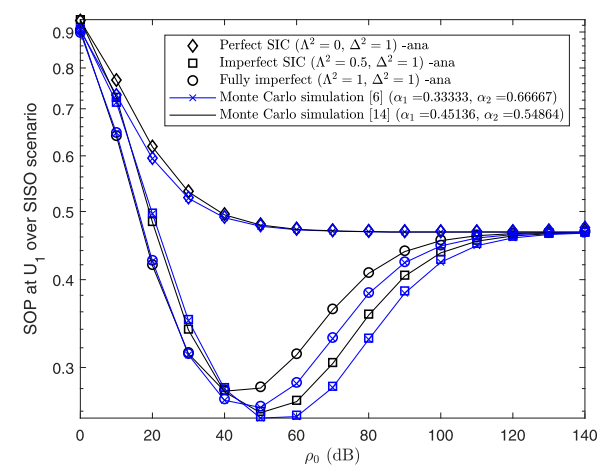

(b)

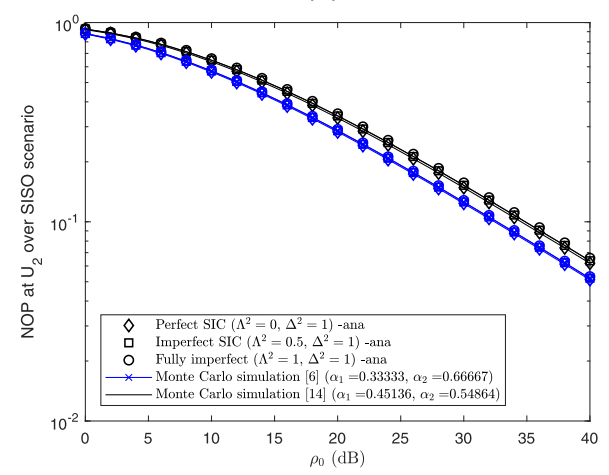

(c)

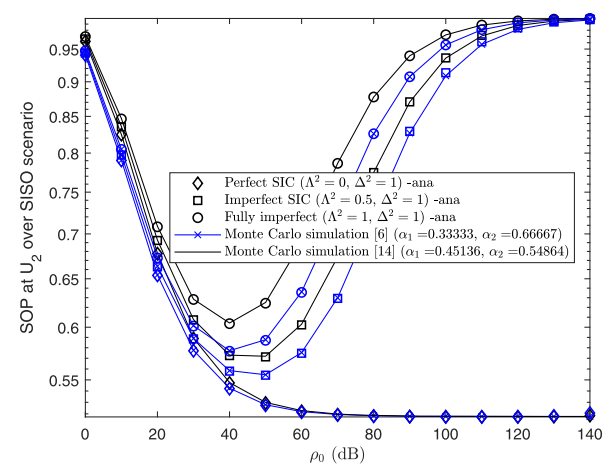

(d)

Fig. 2 a and $\mathbf{b}$ are with NOP and SOP at U1 based on perfect, imperfect, and fully imperfect SIC. c and $\mathbf{d}$ are with NOP and SOP at $U_{2}$ based on perfect, imperfect, and fully imperfect SIC increased, e.g. SNR $\rho_{0}=60 \mathrm{~dB}$. In summary, the SOP results obtained at $U_{1}$ assuming perfect/imperfect/fully imperfect SIC were approximately together at SNRs $\rho_{0} \rightarrow$ $\infty$.

In other investigations, Fig. $2 \mathrm{c}$ and $\mathrm{d}$ plot NOP and SOP results obtained at $U_{2}$ based on two PA strategies by [6, 14]. Due to $\alpha_{2}^{[6]}=0.66667$ given by [6] bigger than $\alpha_{2}^{[14]}=$ 0.54864 given by [14], the obtained NOP results from [6] indicated with blue markers outperform the results indicated with black markers obtained from [14] at SNRs $\rho_{0}$. In addition, the NOP results obtained at $U_{2}$ with the same PA strategy were approximately obtained together based on all three perfect, imperfect and fully imperfect SICs. By observation, equation (38) for $\left(\Lambda^{2}=0\right),\left(\Lambda^{2}=0.5\right)$, and $\left(\Lambda^{2}=1\right)$, we noted that $U_{1}$ was allocated a small power factor $\alpha_{1}^{[6]}=0.33333$ or $\alpha_{1}^{[14]}=0.45136$ because the channel conditions for $U_{1}$ was better than channel conditions for $U_{2}$. The $x_{1}$ symbol therefore lightly impacted when $U_{2}$ decoded its own $x_{2}$ symbol by treating the $x_{1}$ symbol as noise.

Figure $2 \mathrm{~d}$ plots the SOP results at $U_{2}$ assuming perfect/imperfect/fully imperfect SICs. The difference to Fig. $2 \mathrm{~b}$ is easily seen. Assuming perfect $\operatorname{SIC}\left(\Lambda^{2}=0\right)$, the results obtained at $U_{2}$ were the best results compared to other SICs because $U_{2}$ implemented perfect SIC with no impact from internal interference $x_{1}$. Assuming imperfect $\left(\Lambda^{2}=0.5\right)$ and fully imperfect $\left(\Lambda^{2}=1\right)$ SICs at $U_{2}$ because of the impact from internal interference $x_{1}$ and external eavesdropper $E, U_{2}$ 's SOP results from (38) therefore obtained $\Theta_{1}^{(\mathrm{SISO})} \rightarrow 1$ as the secrecy instantaneous bit rate reached $\tilde{R}_{2-x_{2}}^{(\Psi)} \rightarrow 0$ with SNRs $\rho_{0} \rightarrow \infty$. It is worth noting that the results of analysis given by (38) plotted with various markers were proved and verified using Monte Carlo simulation results given by (37) and plotted with black solid and blue crossed-solid lines.

\subsection{Results and discussions for the NOP and SOP}

From the analysis and simulation results obtained in the previous subsection 4.1, we observed that fully imperfect SIC showed a balanced QoS between $U_{2}$ and $U_{1}$. The investigations are therefore discussed below assuming fully imperfect $\left(\Lambda^{2}=1\right)$ at the users.

Figure 3 a plots the NOP results at $U_{1}$ over the three SISO, MISO and MIMO scenarios in four investigations as follows: a SISO scenario equipped with a single antenna at the BS and users $(S=U=1)$; a MISO scenario equipped with a double transmitter antenna at the BS $(S=2)$ and a single receiver antenna at the users $(U=1)$; another MISO scenario equipped with a triple transmitter antenna at the BS $(S=3)$ and a single receiver antenna at the users $(U=1)$; and a MIMO scenario equipped with a double antenna at the BS and users $(S=U=2)$. From the analysis and simulations results, the NOMA system performance progressively improved by equipping more 


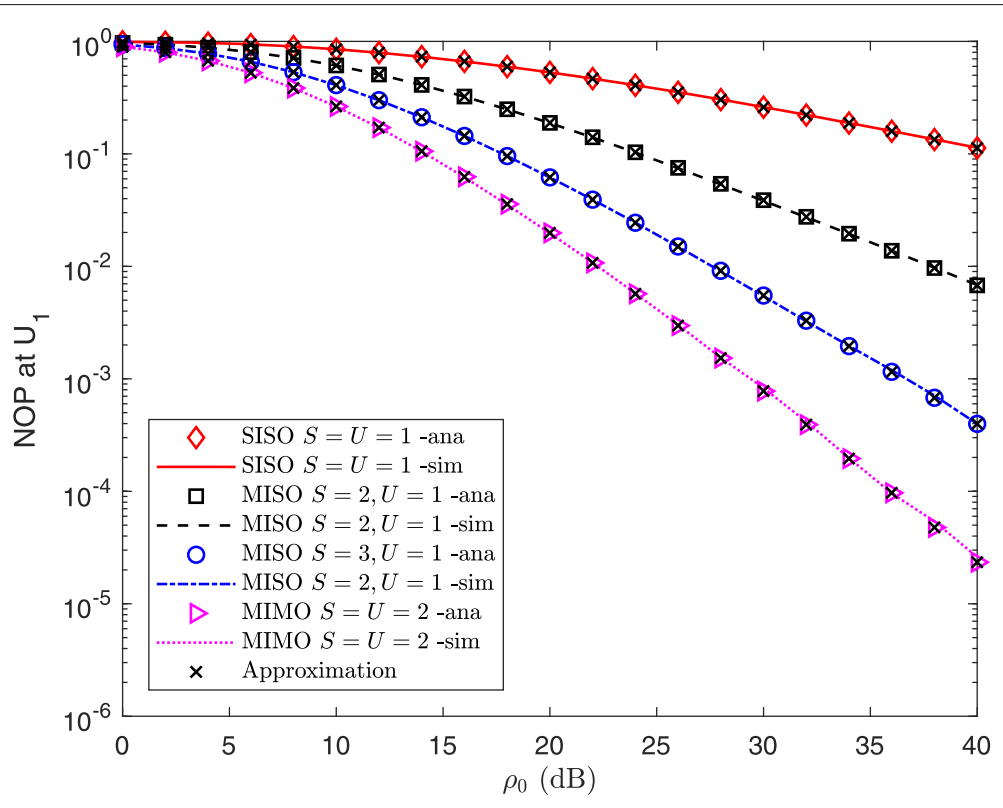

(a)

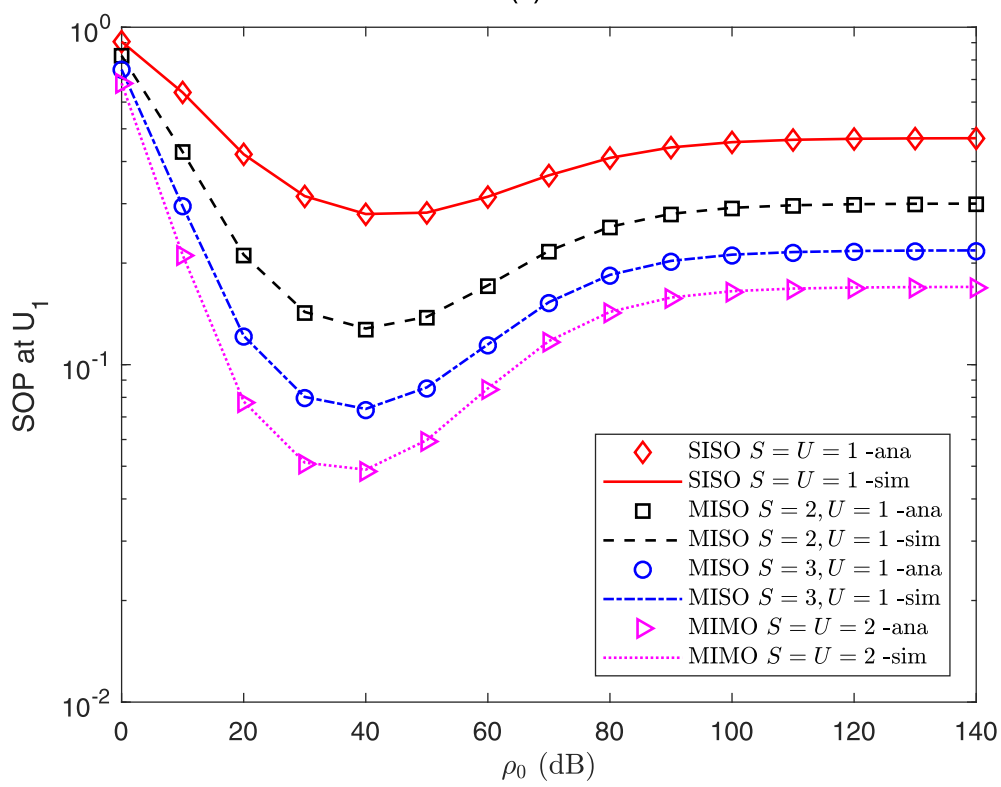

(b)

Fig. 3 a is the NOP at $U_{1}$ over SISO, MISO and MIMO scenarios, and $\mathbf{b}$ is the SOP at $U_{1}$ over individual scenario

antennae. For example, the MISO scenario with $S=3$ obtained better performance than the MISO scenario with $S=2$. However, it was interesting when the MIMO system with $S=2$ obtained better performance than the MISO system with $S=3$. For clarity, the MISO scheme with $S=3$ and $U=1$ had three channels from the BS to each user, while the MIMO scenario with $S=2$ and $U=2$ had four channels from the BS to each user. The TAS protocols over the MIMO scheme selected the best channel from four channels, while only three channels over the
MISO scheme. The different markers plot the results of analysis given by (17), (21) and (27) for the SISO, MISO and MIMO scenarios, respectively. The different crossed markers plot the approximated results given by (22) and (28). The other lines indicate the Monte Carlo simulation results. The analysis and approximated results are generally close and were verified by the Monte Carlo simulation results. Monte Carlo simulations were investigated based on the statistical results of $10^{6}$ experimental iterations, as in previous studies. The analysis, approximation 
and simulation results matched closely, as shown in Fig. 3a.

In addition, Fig. $3 \mathrm{~b}$ plots the SOP results at $U_{1}$ over three individual schemes with the same parameters as Table 1. However, this investigation included an eavesdropper $E$. It is easy to observe that the diamond markers in Fig. $3 \mathrm{~b}$ are close to the SOP results at $U_{2}$ based on fully imperfect SIC, plotted as circle markers in Fig. 2b. The initial impact of an eavesdropper on system performance was negligible, e.g. at low SNRs $\rho_{0} \leq 40 \mathrm{~dB}$, because the eavesdropper found it difficult to successfully decode the $x_{1}$ symbol at $U_{1}$ when it eavesdropped $U_{1}$. As the SNRs $\rho_{0} \rightarrow \infty$ increased, the system performance deteriorated because the eavesdropper $E$ easily decoded the $x_{1}$ symbol at $U_{1}$. In all investigations, the MIMO scenario with $S=U=2$ obtained better results than the other scenarios. The SOP analysis results were plotted with various markers given by (38), (42) and (46) for the SISO, MISO and MIMO scenarios, respectively, while the various lines plot the Monte Carlo simulation results

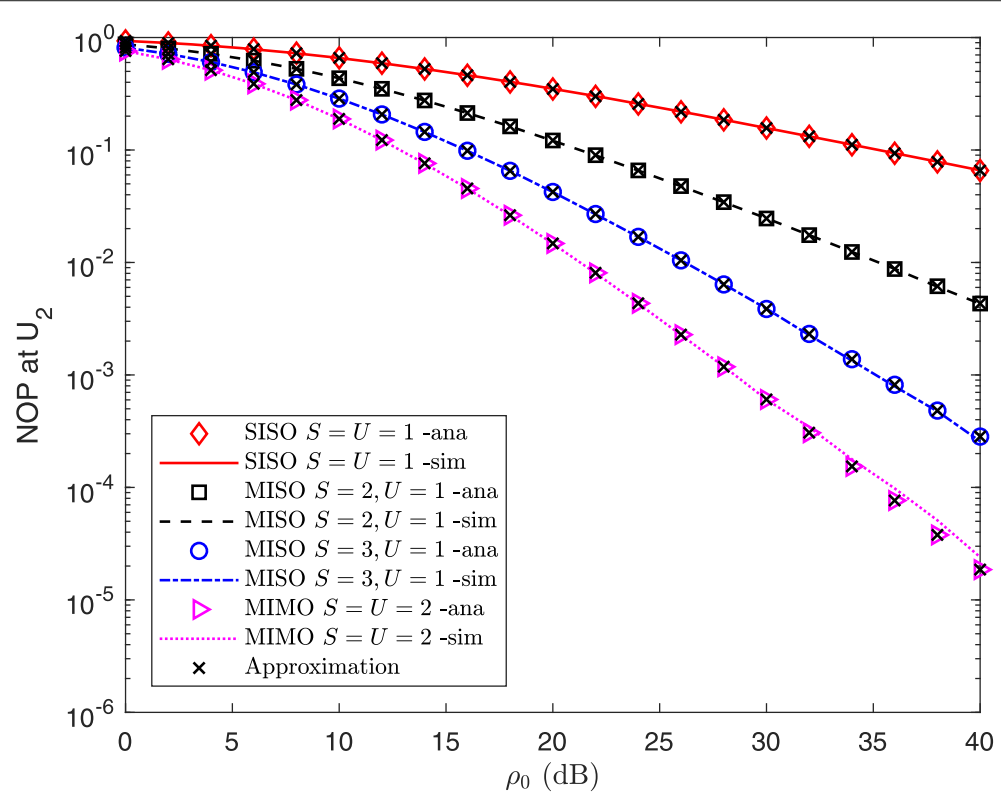

(a)

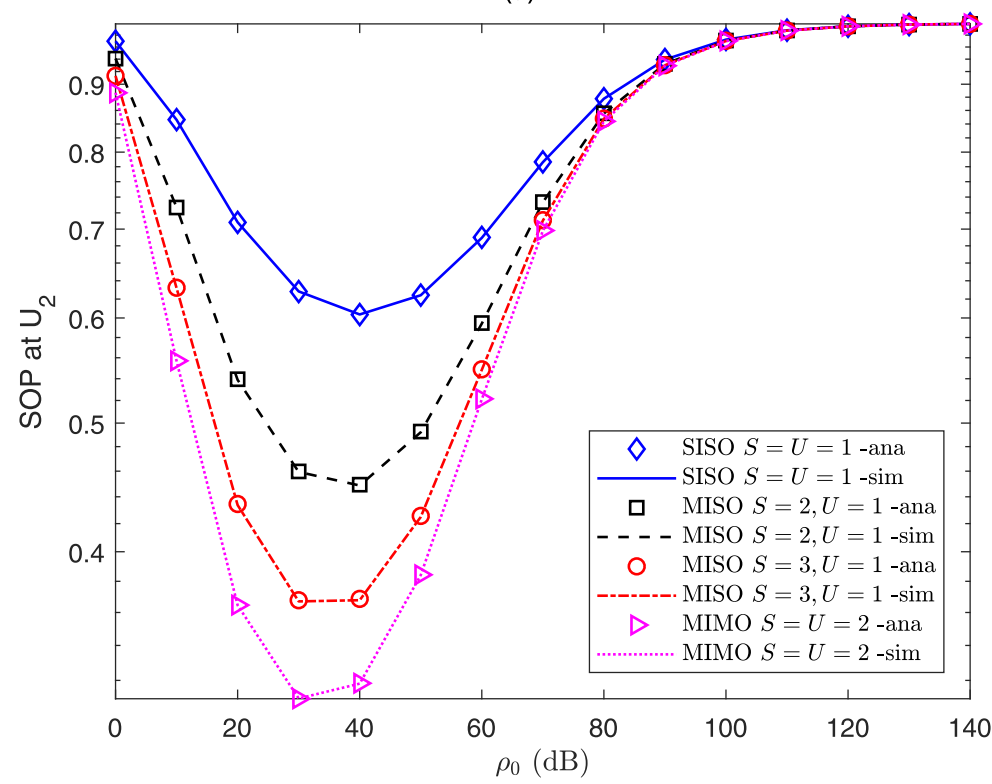

(b)

Fig. 4 a is the NOP results at $U_{2}$ over SISO, MISO and MIMO scenarios, and $\mathbf{b}$ is the SOP results at $U_{2}$ over also three individual scenarios 
given by (37), (41) and (45). Monte Carlo simulations, in particular, were investigated based on the statistical results of $10^{7}$ experimental iterations instead of only $10^{6}$ experimental iterations as in previous investigation, see Fig. 3a. Due to the existence of the eavesdropper $E$, statistics over only $10^{6}$ samples were not guaranteed to be accurate.

This paper also investigated the NOP and SOP at $U_{2}$ over three individual SISO, MISO and MIMO scenarios as shown in Figs. 4a and b, respectively. The NOP and
SOP results at $U_{2}$ were indicated with diamond markers in Fig. 4a and b, the results assuming fully imperfect SIC, plotted with circle markers as shown in Fig. 2c and d. QoS at $U_{2}$ over the MIMO scenario significantly improved compared to its results in other scenarios. Figure 4a plots various markers for NOP analysis results at $U_{2}$ given by (19), (24) and (30) for SISO, MISO and MIMO scenarios, respectively, while crossed markers show the approximated results given by (25) and (31). The various lines plot the Monte Carlo simulation results given by (18), (23)

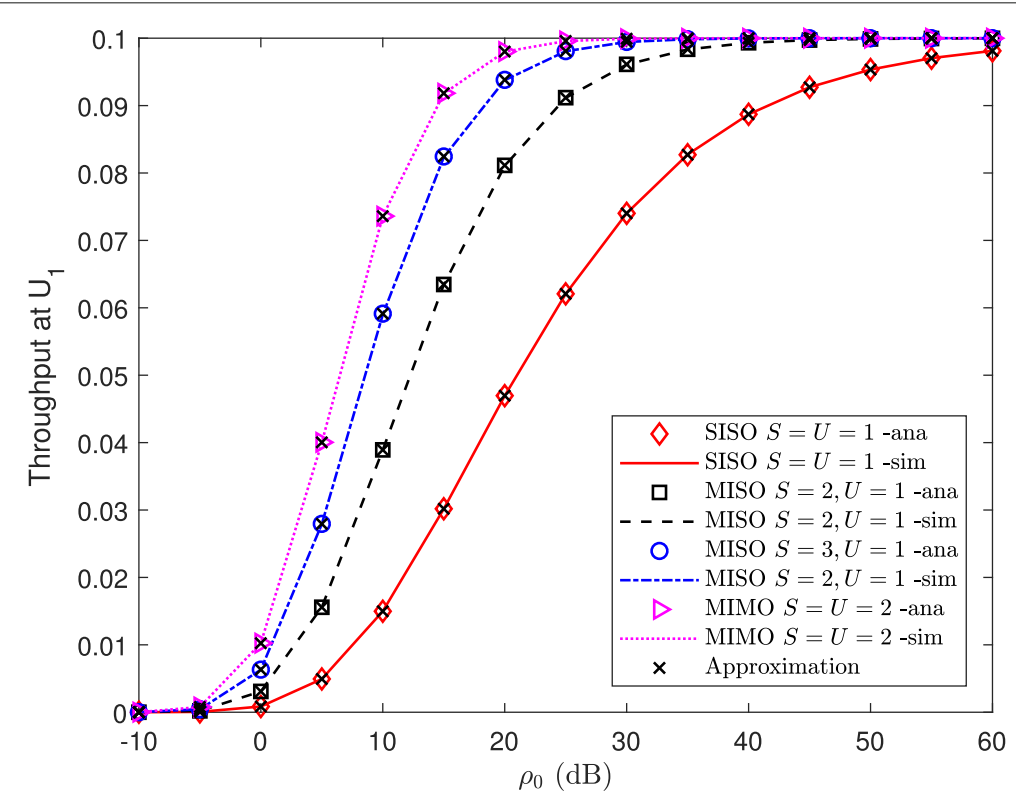

(a)

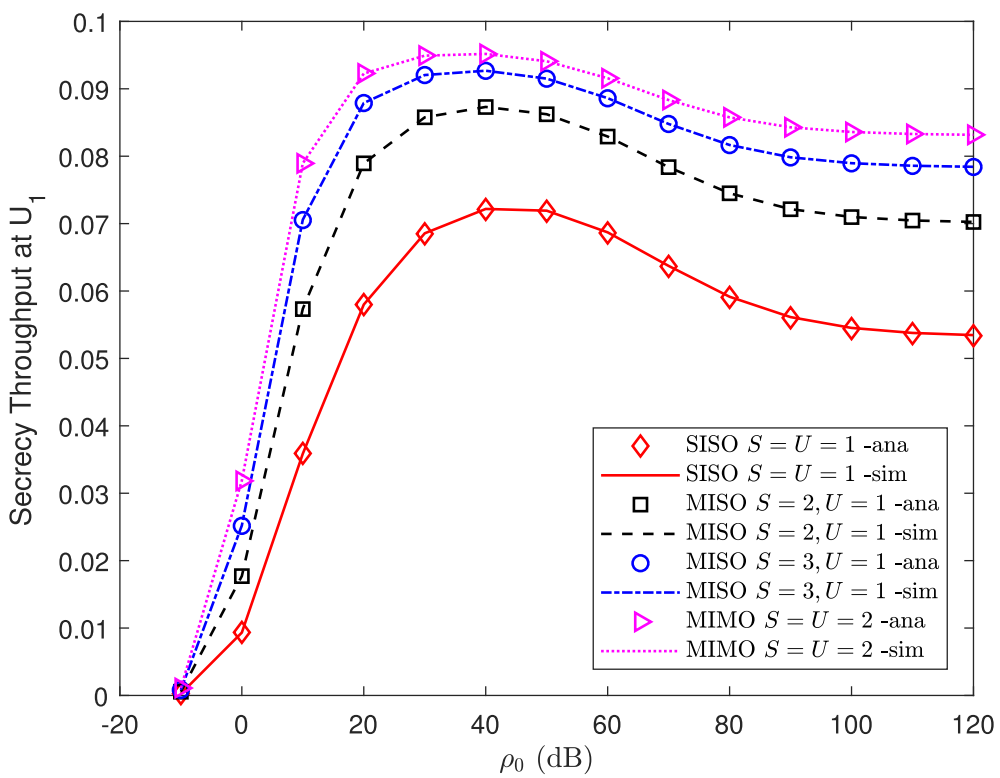

(b)

Fig. 5 a is the throughput of $U_{1}$ over SISO, MISO and MIMO scenarios, and $\mathbf{b}$ is the secure throughput of $U_{1}$ over three individual scenarios 
and (29), respectively. The NOP results at $U_{2}$ obtained $\Theta_{2}^{(\Psi)} \rightarrow 0$ when $\rho_{0} \rightarrow \infty$.

Figure $4 \mathrm{~b}$ shows the plotted results for SOP at $U_{2}$ over three individual scenarios and indicate that the MIMO scenario significantly improved QoS at $U_{2}$ at low SNRs, e.g. $\rho_{0}<40 \mathrm{~dB}$. As SNRs $\rho_{0}$ increased to $40 \mathrm{~dB}$ and over, the SOP performance at $U_{2}$ over all three scenarios developed progressively worse results, e.g. SNRs $\rho_{0} \geq 40$ $\mathrm{dB}$, and then approximated each other for SNRs $\rho_{0} \geq$ $100 \mathrm{~dB}$. At high SNRs, e.g. $\rho_{0}>100 \mathrm{~dB}$, SOP at $U_{2}$ over the three individual scenarios tended to $100 \%$ outage $\left(\tilde{\Theta}_{2}^{(\Psi)} \rightarrow 1\right)$ as a result of the secrecy instantaneous bit rate threshold tending to zero $\left(\tilde{R}_{2-x_{2}}^{(\Psi)} \rightarrow 0\right)$ and the instantaneous bit rate threshold of the eavesdropper $E$ tending to $1\left(R_{E-x_{2}}^{(\Psi)} \rightarrow 1\right)$ when it eavesdropped $U_{2}$ with SNRs $\rho_{0} \rightarrow \infty$. The results of analysis for the three individual scenarios plotted by various markers given by (40), (44) and (48) were also proved and verified using Monte Carlo simulation results given by (39), (43) and (47), plotted as various lines.

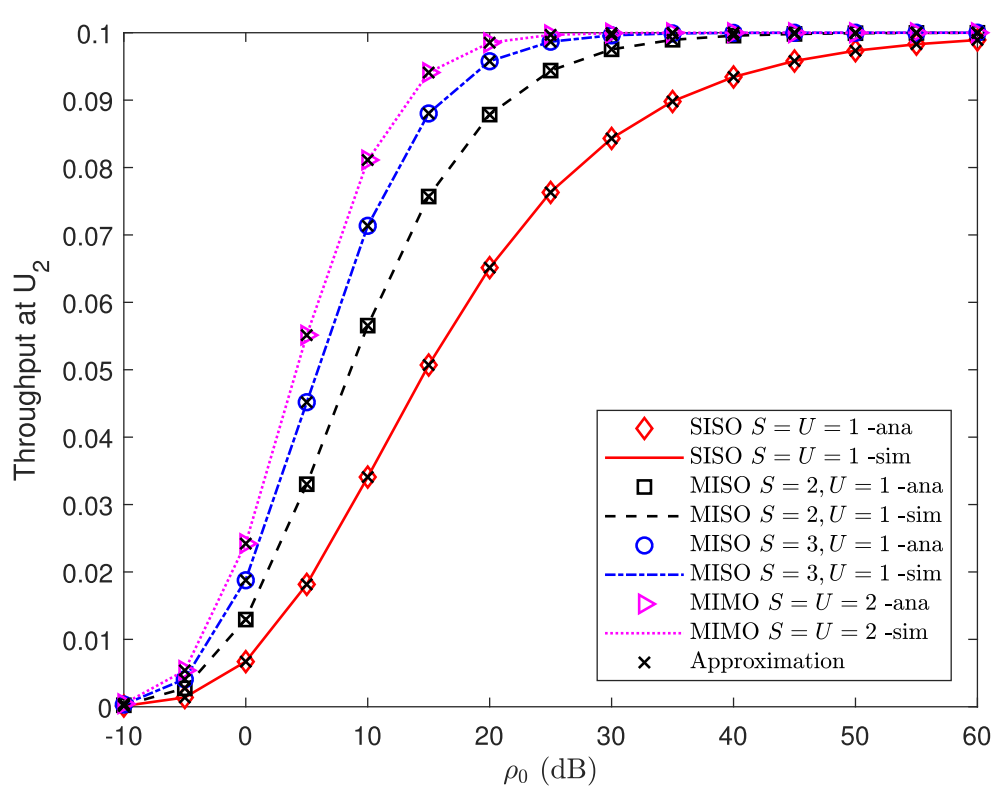

(a)

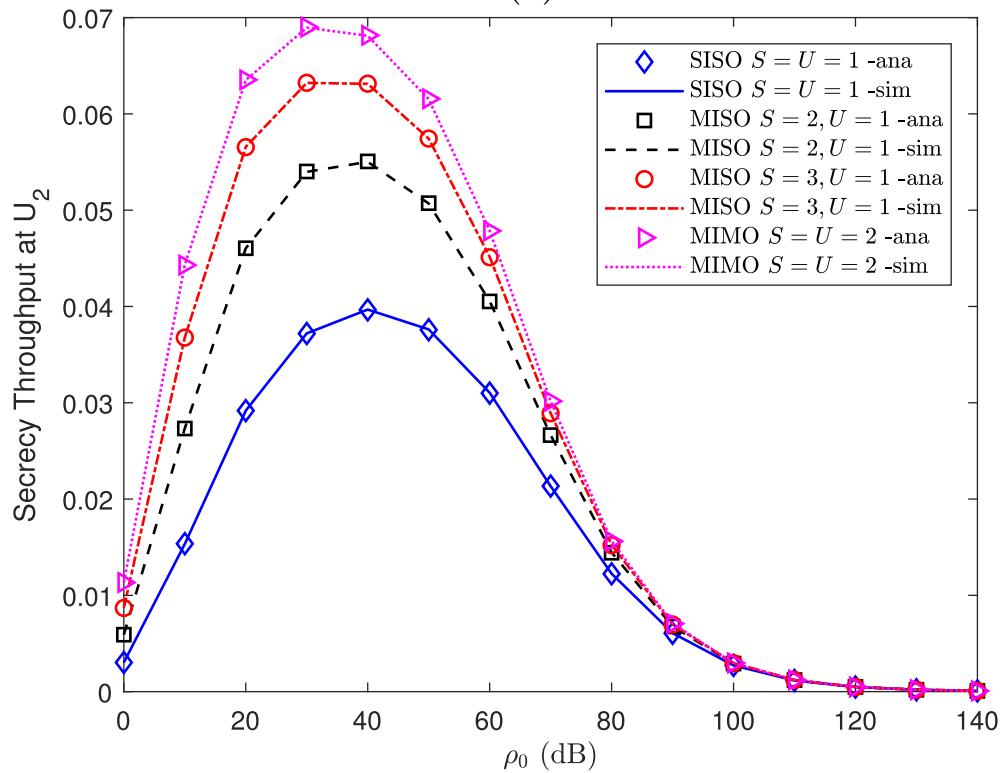

(b)

Fig. $\mathbf{6}$ a is the non-secure throughput of $U_{1}$ over SISO, MISO and MIMO scenarios, and $\mathbf{b}$ is the secure throughput of individual scenarios 


\subsection{Results and discussions for the system throughput} From the results obtained for NOP and SOP at $U_{1}$ and $U_{2}$ over three individual scenarios, we plotted the achievable throughput and secrecy throughput for both $U_{1}$ and $U_{2}$, shown in Figs. $5 \mathrm{a}, \mathrm{b}$ and $6 \mathrm{a}, \mathrm{b}$. Figure $5 \mathrm{a}$ and $\mathrm{b}$ plot the throughput and secure throughput of $U_{1}$ over three individual scenarios, while Figs. $6 \mathrm{a}$ and $\mathrm{b}$ plot the throughput and secure throughput of $U_{2}$ over the same scenarios. We can easily see that the throughput results achieved at $U_{i}$ over the MIMO scenario were slightly better, because the NOP results at $U_{i}$ over the MIMO scenario obtained better results than other scenarios. As SNR $\rho_{0} \rightarrow \infty$ increased, the NOP results therefore tended approximately to zero $\left(\Theta_{i}^{(\Psi)} \rightarrow 0\right)$, as shown in Figs. 3a and 4a. Throughput therefore tended to the user's bit rate threshold $R_{i}^{(*)}=0.1$ bit per channel user (BPCU), as shown in Figs. $5 \mathrm{a}$ and $6 \mathrm{a}$.
Figures $5 \mathrm{~b}$ and $6 \mathrm{~b}$ plot the secrecy throughput of $U_{i}$ over the three individual scenarios. However, it was interesting to observe in Fig. $6 \mathrm{~b}$ that secrecy throughput improved at $U_{2}$ as $\mathrm{SNR} \rho_{0} \rightarrow 40 \mathrm{~dB}$ increased and thereafter reduced to approximately zero instead of tending toward its bit rate threshold $R_{i}^{(*)}$, as in Figs. 5a and b. Because of the eavesdropper $E$, the approximately obtained instantaneous bit rate threshold at $U_{2}$ for SNRs $\rho_{0} \rightarrow \infty$, the SOP results at $U_{2}$ over three individual scenarios tended to $100 \%$ outage $\left(\tilde{\Theta}_{2}^{(\Psi)} \rightarrow 1\right)$ while the secrecy bit rate threshold at $U_{2}$ therefore tended to zero $\left(\tilde{R}_{2-x_{2}}^{(\Psi)} \rightarrow 0\right)$.

\subsection{Results and discussions for the impacts of antennae}

In this section, the impact of antennae on system performance is investigated. All the parameters of investigation in Table 1 were reapplied, however, with fixed SNRs $\rho_{0}=$ $\{20,50\} \mathrm{dB}$.

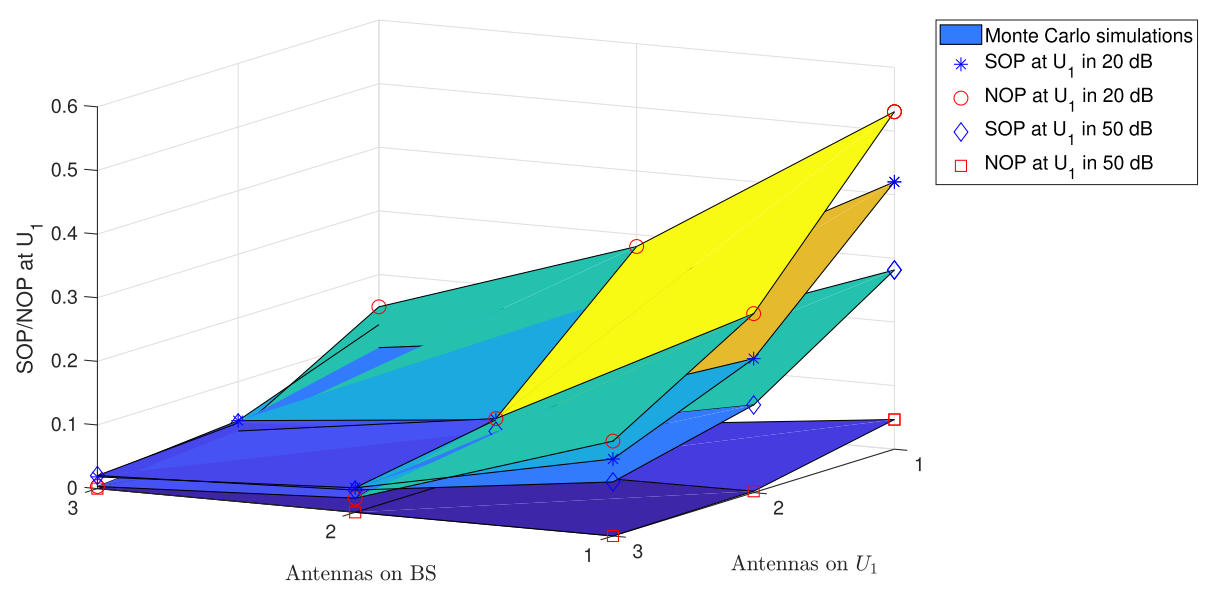

(a)

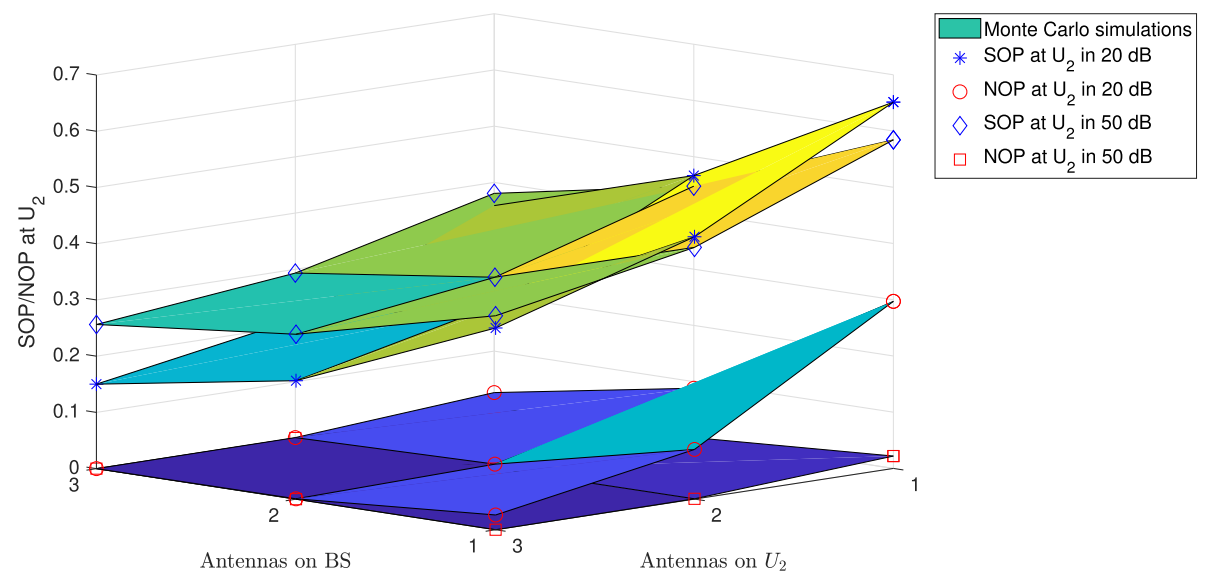

(b)

Fig. 7 The impacts of antennae on SOP/NOP at $\mathbf{a} U_{1}$, and $\mathbf{b} U_{2}$ 
Figure $7 \mathrm{a}$ and $\mathrm{b}$ plot the SOP and NOP results at $U_{1}$ and $U_{2}$, respectively. As a contribution, it is worth noting that the $\mathrm{BS}, U_{1}$ and $U_{2}$ were equipped with multi-antenna technology differentiated from each other instead of being equipped with the same number of antennae as in the previous studies [23] with $M=N=3$ or [24] with $M=N=2$, where $M$ and $N$ denote the number of antennae at the BS and users, respectively. By observation, we can conclude that the system performance improved by equipping more antennae at either the BS or user or both. In addition, the $\mathrm{SNR} \rho_{0}$ also significantly impacted the system's performance. For example, the NOP and SOP results at users obtained with SNR $\rho_{0}=50 \mathrm{~dB}$ outperformed the results at SNR $\rho_{0}=20 \mathrm{~dB}$. Figure $8 \mathrm{a}$ and $\mathrm{b}$ plot the throughput and secrecy throughput results at the users over the three individual scenarios based on the results as shown in Figs. $7 \mathrm{a}$ and $\mathrm{b}$.

\section{Conclusion}

In this paper, a MINO-NOMA system was proposed equipping multiple antennae not only at the BS but also at all users. The TAS protocol was also deployed. An analysis and approximation of NOP and SOP at the users were investigated and the results obtained were expressed in closed form, which were proved and verified using Monte Carlo simulation results based on $10^{6}$ random samples of experiments. In the SOP results, the secrecy system performance was impacted because of an eavesdropper. However, the analysis, approximation and simulation results indicated that secrecy system performance can be significantly enhanced by increasing the number of antennae or the SNRs. This paper therefore demonstrated that multiple antennae combined with the TAS protocol and reasonable PA were an effective strategy for improving secrecy system performance and resisting eavesdropping.

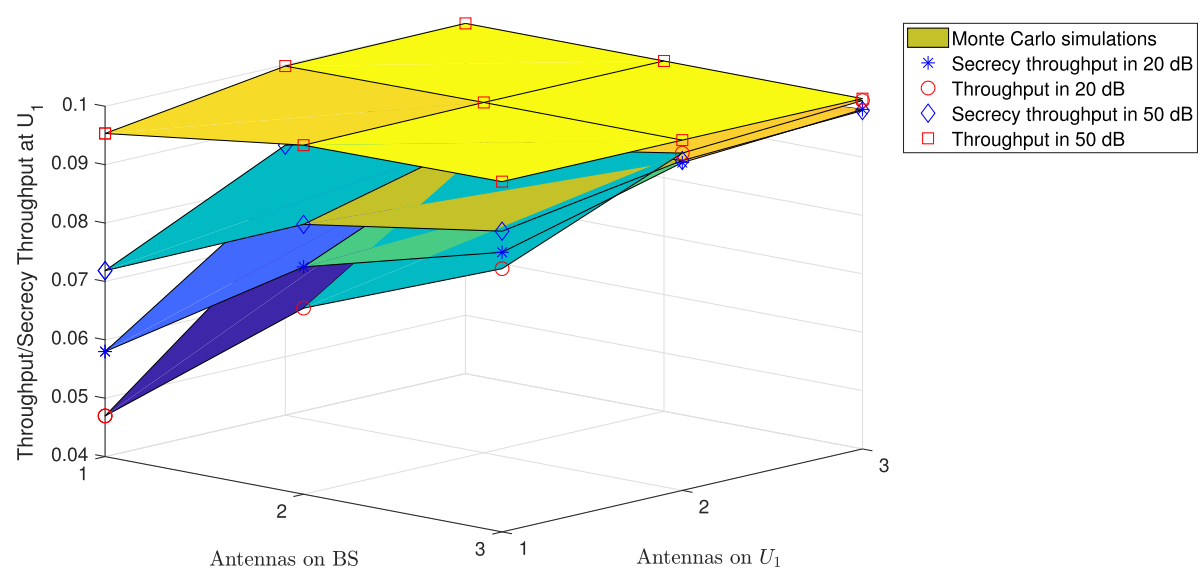

(a)

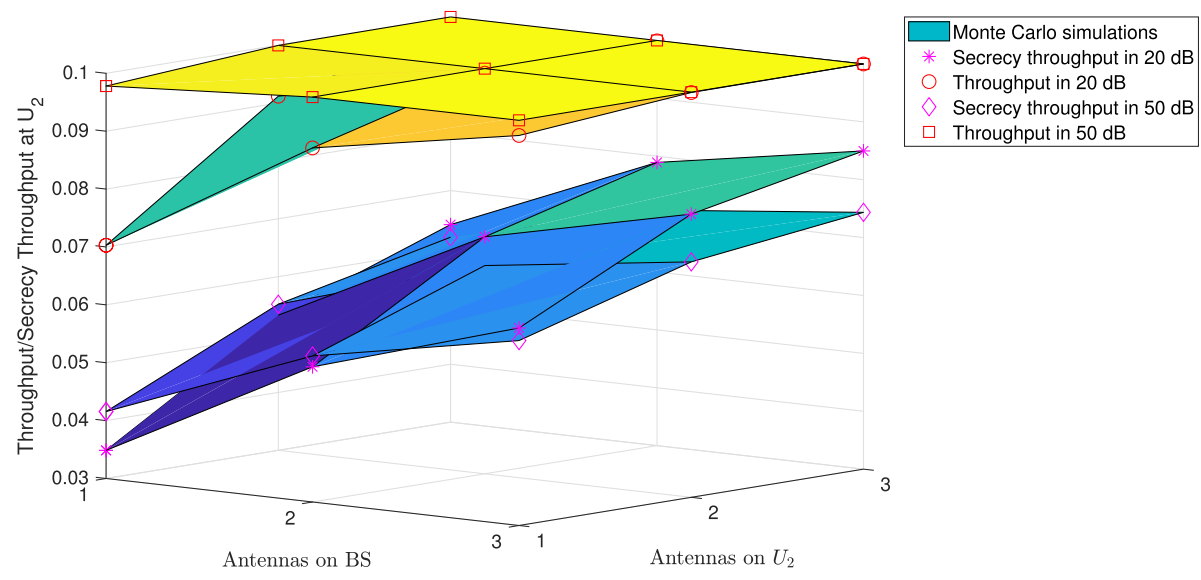

(b)

Fig. 8 The impacts of antennae on system throughput/secrecy throughput at $\mathbf{a} U_{1}$, and $\mathbf{b} U_{2}$ 


\section{Appendix}

\section{The proof of Remark 1}

The CDF of $\gamma_{i-x_{j}}^{(\mathrm{SIO})}$ where $i=\{1,2, E\}$ and $j=\{2,1\}$ can be respectively expressed as follows:

$$
F_{\gamma_{i-x_{j}}^{(\text {SISO) }}}(x) \stackrel{(j=2)}{=} \begin{cases}1-e^{-\frac{x}{\left(\Delta^{2} \alpha_{2}-\Lambda^{2} \alpha_{1} x\right) \rho_{0} \sigma_{0, i}^{2}},} & \text { where }\left(x<\frac{\Delta^{2} \alpha_{2}}{\Lambda^{2} \alpha_{1}}\right), \\ 1, & \text { where }\left(x \geq \frac{\Delta^{2} \alpha_{2}}{\Lambda^{2} \alpha_{1}}\right),\end{cases}
$$

and,

$$
F_{\gamma_{i-x_{j}}(\text { SISO) }}(x) \stackrel{(j=1)}{=} 1-e^{-\frac{x}{\Lambda^{2} \alpha_{1} \rho_{0} \sigma_{0, i}^{2}}}, \text { where }(x \geq 0) .
$$

However, the PDF of $\gamma_{i-x_{j}}^{\text {(SISO) }}$ where $i=\{1,2, E\}$ and $j=$ $\{2,1\}$ can be also respectively expressed as follows:

$$
\begin{aligned}
& f_{\gamma_{i-x_{j}} \text { (SISO) }}(x) \\
& \stackrel{(j=2)}{=} \begin{cases}\frac{\Delta^{2} \alpha_{2}}{\left(\Delta^{2} \alpha_{2}-\Lambda^{2} \alpha_{1} x\right)^{2} \rho_{0} \sigma_{0, i}^{2}} e^{-\frac{x}{\left(\Delta^{2} \alpha_{2}-\Lambda^{2} \alpha_{1} x\right) \rho_{0} \sigma_{0, i}^{2}}} & \text { where }\left(x<\frac{\Delta^{2} \alpha_{2}}{\Lambda^{2} \alpha_{1}}\right), \\
0, & \text { where }\left(x \geq \frac{\Delta^{2} \alpha_{2}}{\Lambda^{2} \alpha_{1}}\right),\end{cases}
\end{aligned}
$$

and

$$
f_{\gamma_{i-x_{j}}^{\text {(SISO) }}}(x) \stackrel{(j=1)}{=} \frac{1}{\Lambda^{2} \alpha_{1} \rho_{0} \sigma_{0, i}^{2}} e^{-\frac{x}{\Lambda^{2} \alpha_{1} \rho_{0} \sigma_{0, i}^{2}}}, \text { where }(x \geq 0) .
$$

By substituting (2) or (3) into (4) and combining with the outage conditions in (16) or (18), we obtain the expressions as follows:

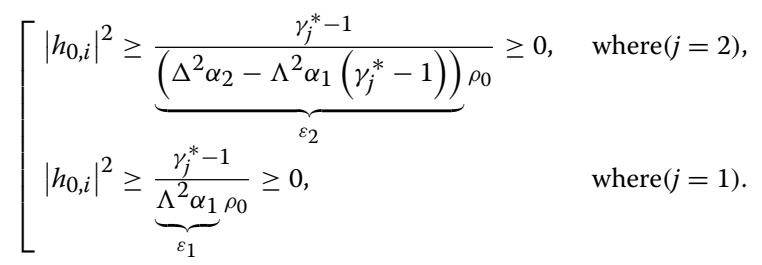

By applying the PDF, the NOP at $U_{i}$ for $i=\{1,2\}$ obtained when it cannot successfully detect the $x_{j}$ symbol for $j=\{2,1\}$ is expressed as

$$
\begin{aligned}
\Theta_{i-x_{j}}^{(\Psi)} & =\operatorname{Pr}\left\{R_{i-x_{j}}^{(\mathrm{SISO})} \geq R_{j}^{*}\right\}=\int_{\frac{\gamma_{j}^{*}-1}{\varepsilon_{j} \rho_{0}}}^{\infty} \frac{1}{\sigma_{0, i}^{2}} e^{-\frac{x}{\sigma_{0, i}^{2}}} d x \\
& \stackrel{(j=2)}{=} \int_{\gamma_{2}^{*}-1}^{\infty} \frac{\Delta^{2} \alpha_{2}}{\left(\Delta^{2} \alpha_{2}-\Lambda^{2} \alpha_{1} x\right)^{2} \rho_{0} \sigma_{0, i}^{2}} e^{-\frac{x}{\left(\Delta^{2} \alpha_{2}-\Lambda^{2} \alpha_{1} x\right) \rho_{0} \sigma_{0, i}^{2}}} d x \\
& =e^{-\frac{\gamma_{2}^{*}-1}{\varepsilon_{2} \rho_{0} \sigma_{0, i}^{2}}}
\end{aligned}
$$

$$
\stackrel{(j=1)}{=} \int_{\gamma_{1}^{*}-1}^{\infty} \frac{1}{\Lambda^{2} \alpha_{1} \rho_{0} \sigma_{0, i}^{2}} e^{-\frac{x}{\Lambda^{2} \alpha_{1} \rho_{0} \sigma_{0, i}^{2}}} d x=e^{-\frac{\gamma_{1}^{*}-1}{\varepsilon_{1} \rho_{0} \sigma_{0, i}^{2}}}
$$

By substituting (55) and (56) for $i=1$ and $j=\{2,1\}$ into (16), we easily obtain the closed form of the NOP at $U_{1}$ over SISO scheme as shown in (17). Similarly, the NOP at $U_{2}$ over SISO scheme can be obtained in closed form as shown in (19) by substituting (55) for $i=j=2$ into (18).

\section{The proof of Remark 2}

The CDF of $\gamma_{i-x_{j}}^{(\mathrm{MISO})}$, where $i=\{1,2, E\}, j=\{2,1\}$ and $s=[1 \cdots S]$, can be respectively expressed as follows:

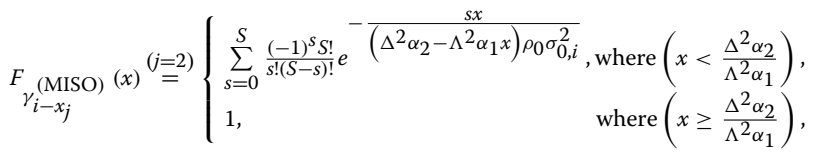

and

$$
F_{\gamma_{i-x_{j}}^{(\mathrm{MISO})}}(x) \stackrel{(j=1)}{=} \sum_{s=0}^{S} \frac{(-1)^{s} S !}{s !(S-s) !} e^{-\frac{s x}{\Lambda^{2} \alpha_{1} \rho_{0} \sigma_{0, i}^{2}}} \text {, where }(x \geq 0) \text {. }
$$

However, the PDF of $\gamma_{i-x_{j}}^{(\mathrm{MISO})}$ for $i=\{1,2, E\}$ and $j=$ $\{2,1\}$ is expressed as follows:

$$
\begin{aligned}
& { }_{\gamma_{i-x_{j}}^{\text {(MISO) }}}^{(x)} \\
& \stackrel{(=2)}{=}\left\{\begin{array}{lr}
\prod_{s=1}^{S} \frac{\Delta^{2} \alpha_{2}}{\left(\Delta^{2} \alpha_{2}-\Lambda^{2} \alpha_{1} x\right)^{2} \rho_{0} \sigma_{0, i}^{2}} e^{-\frac{x}{\left(\Delta^{2} \alpha_{2}-\Lambda^{2} \alpha_{1} x\right) \rho_{0} \sigma_{0, i}^{2}}}, \text { where }\left(x<\frac{\Delta^{2} \alpha_{2}}{\Lambda^{2} \alpha_{1}}\right), \\
0, & \text { where }\left(x \geq \frac{\Delta^{2} \alpha_{2}}{\Lambda^{2} \alpha_{1}}\right), \\
\stackrel{(j=1)}{=} \prod_{s=1}^{S} \frac{1}{\Lambda^{2} \alpha_{1} \rho_{0} \sigma_{0, i}^{2}} e^{-\frac{x}{\Lambda^{2} \alpha_{1} \rho_{0} \sigma_{0, i}^{2}},} & \text { where }(x \geq 0) .
\end{array}\right.
\end{aligned}
$$

By substituting (6) or (7) into (8) and combining with the outage conditions in (20) or (23), we obtain the expression as follows:

$$
\left[\begin{array}{l}
\max \left\{\left[\begin{array}{c}
{\left[\left.h_{0, i}^{(s, 1)}\right|^{2}\right]} \\
s=[1 \cdots S]
\end{array}\right]\right\} \underbrace{\frac{\gamma_{j}^{*}-1}{\left(\Delta^{2} \alpha_{2}-\Lambda^{2} \alpha_{1}\left(\gamma_{j}^{*}-1\right)\right) \rho_{0}}}_{\varepsilon_{2}}, \text { where }(j=2), \\
\max \left\{\left[\begin{array}{c}
\left|h_{0, i}^{(s, 1)}\right|^{2} \\
s=[1 \cdots S]
\end{array}\right]\right\} \geq \underbrace{\frac{\gamma_{j}^{*}-1}{\Lambda^{2} \alpha_{1} \rho_{0}}}_{\varepsilon_{1}}, \quad \text { where }(j=1) .
\end{array}\right.
$$

The NOP at $U_{i}$ for $i=\{1,2\}$ obtained when it cannot successfully decode the $x_{j}$ symbol for $j=\{2,1\}$ expressed as follows: 


$$
\begin{aligned}
\Theta_{i-x_{j}}^{(\mathrm{MISO})} & =\operatorname{Pr}\left\{\max _{s=[1 \cdots S]}\left\{R_{i-x_{j}}^{(s, 1)}\right\}<R_{j}^{*}\right\} \\
& =\prod_{s=1}^{S}\left(1-\int_{\frac{\gamma_{j}^{*}-1}{\varepsilon_{j} \rho_{0}}}^{\infty} \frac{1}{\sigma_{0, i}^{2}} e^{-\frac{x}{\sigma_{0, i}^{2}}} d x\right) \\
& =\prod_{s=1}^{S}\left(1-e^{-\frac{\gamma_{j}^{*}-1}{\varepsilon_{j} \rho_{0} \sigma_{0, i}^{2}}}\right) .
\end{aligned}
$$

By substituting (62), where $i=1$ and $j=\{2,1\}$ into (20), we easily obtain the closed form of the NOP at $U_{1}$ over MISO scheme as shown in (21). Similarly, the NOP at $U_{2}$ over MISO scheme can be also obtained in closed form as shown in (24) by substituting (62), where $i=2$ and $j=2$, into (23).

\section{The proof of Remark 3}

By substituting (11) or (12) into (13) and combining with the outage conditions in (26) or (29), we obtain the expression as follows:

$$
\left[\begin{array}{c}
\max \{\left[\begin{array}{c}
u=[1 \cdots U] \\
{\left[\left|h_{0, i}^{(s, u)}\right|^{2}\right]} \\
s=[1 \cdots S]
\end{array}\right] \geq \underbrace{\frac{\gamma_{j}^{*}-1}{\left(\Delta^{2} \alpha_{2}-\Lambda^{2} \alpha_{1}\left(\gamma_{j}^{*}-1\right)\right)} \rho_{0}}_{\varepsilon_{2}}, \text { where }(j=2), \\
\max \{\left[\begin{array}{c}
u=[1 \cdots U] \\
{\left[\left|h_{0, i}^{(s, u)}\right|^{2}\right]} \\
s=[1 \cdots S]
\end{array}\right] \geq \underbrace{\frac{\gamma_{j}^{*}-1}{\Lambda^{2} \alpha_{1} \rho_{0}},}_{\varepsilon_{1}} \quad \text { where }(j=1) .
\end{array}\right.
$$

The NOP at $U_{i}$ for $i=\{1,2\}$ when it cannot successfully decode the $x_{j}$ symbol for $j=\{2,1\}$ over MIMO scheme is expressed as follows:

$$
\begin{aligned}
\Theta_{i-x_{j}}^{(\mathrm{MISO})} & =\operatorname{Pr}\left\{\max _{s=[1 \cdots S], u=[1 \cdots U]}\left\{R_{i-x_{j}}^{(s, u)} \geq R_{j}^{*}\right\}\right. \\
& =\prod_{s=1}^{S} \prod_{u=1}^{U}\left(\int_{\frac{\gamma_{j}^{*}-1}{\varepsilon_{j} \rho_{0}}}^{\infty} \frac{1}{\sigma_{0, i}^{2}} e^{-\frac{x}{\sigma_{0, i}^{2}}} d x\right) \\
& =\prod_{s=1}^{S} \prod_{u=1}^{U}\left(e^{-\frac{\gamma_{j}^{*}-1}{\varepsilon_{j} \rho_{0} \sigma_{0, i}^{2}}}\right) .
\end{aligned}
$$

By substituting (64), where $i=1$ and $j=\{2,1\}$, into (26), we easily obtain the closed form of the NOP at $U_{1}$ over MIMO scheme as shown in (27). Similarly, the NOP at $U_{2}$ over MIMO scheme can be also obtained in closed form as shown in (30) by substituting (64), where $i=2$ and $j=2$, into (29).

\section{The proof of Remark 4}

By substituting (4) for $i=\{1, E\}$ and $j=1$ into (32) and combining secure outage conditions in (37), we obtain the SOP at $U_{1}$ when it cannot successfully detect $x_{1}$ symbol expressed as follows:

$$
\begin{aligned}
\tilde{\Theta}_{1-x_{1}}^{(\mathrm{SISO})}=\operatorname{Pr}\left\{\max \left\{R_{1-x_{1}}^{(\mathrm{SISO})}-R_{E-x_{1}}^{(\mathrm{SISO})}, 0\right\}<R_{1}^{*}\right\} \\
=\left[\begin{array}{ll}
\operatorname{Pr}\left\{\frac{\left.\gamma_{1-x_{1}}^{(\mathrm{SIO})}<\gamma_{1}^{*}-1\right\},}{\gamma_{E-x_{1}}^{(\mathrm{SISO})}},\right. & \text { where }\left(R_{1-x_{1}}^{\text {(SISO) }}>R_{E-x_{1}}^{(\mathrm{SISO})}\right), \\
1, & \text { where }\left(R_{1-x_{1}}^{(\mathrm{SISO})} \leq R_{E-x_{1}}^{(\mathrm{SISO})}\right) .
\end{array}\right.
\end{aligned}
$$

By applying the PDF, Eq. (65), where $R_{1-x_{1}}^{\text {(SISO) }}>R_{E-x_{1}}^{\text {(SISO) }}$, can be solved and expressed in closed form as follows:

$$
\begin{aligned}
\tilde{\Theta}_{1-x_{1}}^{(\mathrm{SISO})} & =1-\int_{0}^{\infty} \int_{y\left(\gamma_{1}^{*}-1\right)}^{\infty} \frac{1}{\sigma_{0,1}^{2} \sigma_{0, E}^{2}} e^{-\left(\frac{x}{\sigma_{0,1}^{2}}+\frac{y}{\sigma_{0, E}^{2}}\right)} d x d y \\
& =1-\frac{\sigma_{0,1}^{2}}{\gamma_{1}^{*} \sigma_{0, E}^{2}+\sigma_{0,1}^{2}} e^{-\frac{\gamma_{1}^{*}-1}{\Lambda^{2} \alpha_{1} \rho_{0} \sigma_{0,1}^{2}}}
\end{aligned}
$$

Similarly, we obtain the SOP at $U_{i}$ when it cannot successfully detect the $x_{2}$ symbol by substituting (4) into (32) and combining secure outage conditions in (37) or (39) as follows:

$$
\begin{aligned}
\tilde{\Theta}_{i-x_{2}}^{(\mathrm{SISO})}=\operatorname{Pr}\left\{\max \left\{R_{i-x_{2}}^{(\mathrm{SISO})}-R_{E-x_{2}}^{(\mathrm{SISO})}, 0\right\}\right. & \left.<R_{2}^{*}\right\} \\
& =\left[\begin{array}{ll}
\operatorname{Pr}\left\{\frac{\gamma_{i-x_{2}}^{(\mathrm{SIO})}}{\left.\gamma_{E-x_{2}}^{(\mathrm{SISO})}<\gamma_{2}^{*}-1\right\},}\right. & \text { where }\left(R_{i-x_{2}}^{(\mathrm{SISO})}>R_{E-x_{2}}^{(\mathrm{SISO})}\right), \\
1, & \text { where }\left(R_{i-x_{2}}^{(\mathrm{SISO})} \leq R_{E-x_{2}}^{(\mathrm{SISO})}\right) .
\end{array}\right.
\end{aligned}
$$

We attempted to solve (67) using integrals with condition $R_{1-x_{1}}^{(\mathrm{SISO})}>R_{E-x_{1}}^{(\mathrm{SISO})}$. However, it is difficult to obtain the SOP at $U_{i}$ when it cannot decode $x_{2}$ symbol in closed form. The authors in [35] proposed applying the Gaussian-Chebyshev quadrature method to obtain an approximation expression. The SOP at $U_{i}$ when it cannot decode the $x_{2}$ symbol therefore obtained and expressed as follows:

$$
\begin{aligned}
& \tilde{\Theta}_{i-x_{2}}^{\text {(SISO) }} \\
& =1-\frac{\Delta^{2} \alpha_{2}}{\rho_{0} \sigma_{0, E}^{2}} \int_{0}^{\varpi} \frac{1}{\left(\Delta^{2} \alpha_{2}-\Lambda^{2} \alpha_{1} x\right)^{2}} \\
& \times e^{-\left(\frac{\gamma_{2}^{*} x+\gamma_{2}^{*}-1}{\left(\Delta^{2} \alpha_{2}-\Lambda^{2} \alpha_{1}\left(\gamma_{2}^{*}-1\right)-\Lambda^{2} \alpha_{1} \gamma_{2}^{*} x\right) \rho_{0} \sigma_{0, i}^{2}}+\frac{x}{\left(\Delta^{2} \alpha_{2}-\Lambda^{2} \alpha_{1} x\right) \rho_{0} \sigma_{0, E}^{2}}\right)} d x \\
& =1-\frac{\Delta^{2} \alpha_{2} \varpi \pi}{2 \mathrm{~W} \rho_{0} \sigma_{0, E}^{2}} \sum_{w=1}^{W} \frac{\sqrt{1-\lambda^{2}}}{\left(\Delta^{2} \alpha_{2}-\Lambda^{2} \alpha_{1} \beta\right)^{2}} e^{-\theta_{i}}
\end{aligned}
$$




\section{The proof of Remark 5}

By using the CDF (57-58), and PDF (59-60), the SOP at $U_{1}$ is expressed as follows:

$$
\begin{aligned}
& \tilde{\Theta}_{1}^{(\mathrm{MISO})}=\tilde{\Theta}_{1-x_{1}}^{(\mathrm{MISO})} \tilde{\Theta}_{1-x_{2}}^{(\mathrm{MISO})} \\
& =\int_{0}^{\infty} F \gamma_{1-x_{1}}^{\text {(MISO) }}\left(\gamma_{1}^{*}(1+x)-1\right) f_{\gamma_{E-x_{1}}^{(\mathrm{MISO})}}(x) d x \\
& \times\left(\int_{0}^{\sigma} F_{\gamma_{1-x_{2}}^{\text {(MISO) }}}\left(\gamma_{2}^{*}(1+x)-1\right) f_{\gamma_{E-x_{2}}^{\text {(MISO) }}}(x) d x+\int_{\varpi}^{\frac{\Delta^{2} \alpha_{2}}{\Lambda^{2} \alpha_{1}}} f_{\gamma_{E-x_{2}}}^{\text {(MISO) }}(x) d x\right) \\
& =\sum_{s=0}^{S} \frac{(-1)^{s} S ! \sigma_{0,1}^{2}}{s !(S-s) !\left(s \gamma_{1}^{*} \sigma_{0, E}^{2}+\sigma_{0,1}^{2}\right)} e^{-\frac{s\left(\gamma_{1}^{*}-1\right)}{\Lambda^{2} \alpha_{1} \rho_{0} \sigma_{0,1}^{2}}} \\
& \times(\underbrace{\sum_{s=0}^{S} \frac{(-1)^{s} \Lambda^{2} \alpha_{2} S !}{s !(S-s) ! \rho_{0} \sigma_{0, E}^{2}} \int_{0}^{\infty} \frac{1}{\mu^{2}} e^{-\left(\frac{s \Omega}{\left(\Delta^{2} \alpha_{2}-\Lambda^{2} \alpha_{1} \Omega\right) \rho_{0} \sigma_{0, i}^{2}}+\frac{x}{\mu^{2}}\right)} d x}_{G_{i}(i=1)} \\
& \left.+e^{-\frac{\omega}{\left(\alpha_{2}-\alpha_{1} \varpi\right) \rho_{0} \sigma_{0, E}^{2}}}\right)
\end{aligned}
$$

where $\Omega=\gamma_{2}^{*}(1+x)-1$, and $\mu=\Delta^{2} \alpha_{2}-\Lambda^{2} \alpha_{1} x$.

Using CDF (57) and Gaussian-Chebyshev quadrature, $G_{i}$ for $i=\{1,2\}$ can be obtained as follows:

$$
\begin{aligned}
G_{i}= & \sum_{s=0}^{S} \frac{(-1)^{s} \alpha_{2} S !}{s !(S-s) ! \rho_{0} \sigma_{0, E}^{2}} \\
& \times \int_{0}^{\varpi} \frac{1}{\left(\Delta^{2} \alpha_{2}-\Lambda^{2} \alpha_{1} x\right)^{2}} \\
& e^{-\left(\frac{s\left(\gamma_{2}^{*}(1+x)-1\right)}{\left(\Delta^{2} \alpha_{2}-\Lambda^{2} \alpha_{1}\left(\gamma_{2}^{*}(1+x)-1\right)\right) \rho_{0} \sigma_{0, i}^{2}}+\frac{x}{\left(\Delta^{2} \alpha_{2}-\Lambda^{2} \alpha_{1} x\right)^{2}}\right)} d x \\
= & \frac{\varpi \pi \Delta^{2} \alpha_{2} S !}{2 \rho_{0} W \sigma_{0, E}^{2}} \sum_{s=0}^{S} \sum_{w=1}^{W} \frac{(-1)^{s} \sqrt{1-\lambda^{2}} e^{-\Phi_{i}}}{s !(S-s) !\left(\Delta^{2} \alpha_{2}-\Lambda^{2} \alpha_{1} \beta\right)^{2}} .
\end{aligned}
$$

By substituting (70) into (69), we obtained the closed form of the SOP at $U_{1}$ over the MISO scheme as shown in(42). From (70), we can also obtain the closed form of the SOP at $U_{2}$ over the MISO scheme as shown in the (44) with outage conditions as shown in (43).

\section{The proof of Remark 6}

From (57), the CDF of $\gamma_{i-x_{2}}^{(\mathrm{MIMO})}$ was rewritten and expressed as follows:

$F_{\gamma_{i-x_{2}}(\mathrm{MIMO})}= \begin{cases}\sum_{n=0}^{N=S U} \frac{(-1)^{n}(N) !}{n !(N-n) !} e^{-\frac{n x}{\left(\Delta^{2} \alpha_{2}-\Lambda^{2} \alpha_{1} x\right) \rho_{0} \sigma_{0, i}^{2}}}, & \text { where }\left(x<\frac{\Delta^{2} \alpha_{2}}{\Lambda^{2} \alpha_{1}}\right), \\ 1, & \text { where }\left(x \geq \frac{\Delta^{2} \alpha_{2}}{\Lambda^{2} \alpha_{1}}\right) .\end{cases}$

The SOP at $U_{1}$ is expressed as follows:

$$
\begin{aligned}
& \tilde{\Theta}_{1}^{(\mathrm{MIMO})}=\tilde{\Theta}_{1-x_{1}}^{(\mathrm{MIMO})} \tilde{\Theta}_{1-x_{2}}^{(\mathrm{MIMO})} \\
& =\int_{0}^{\infty} F_{1-x_{1}}^{(\text {MIMO) }}\left(\gamma_{1}^{*}(1+x)-1\right) f_{\gamma_{E-x_{1}}^{(\mathrm{MIMO})}(x) d x} \\
& \times\left(\int_{0}^{\infty} F_{1-x_{2}}^{\text {(MIMO) }}\left(\gamma_{2}^{*}(1+x)-1\right)\right. \\
& \left.f_{E-x_{2}}^{(\mathrm{MIMO})}(x) d x+\int_{\sigma}^{\frac{\Delta^{2} \alpha_{2}}{\Lambda^{2} \alpha_{1}}} f_{E-x_{2}}^{(\mathrm{MIMO})}(x) d x\right) \\
& =\sum_{n=0}^{N=S U} \frac{(-1)^{n} N ! \sigma_{0,1}^{2}}{n !(N-n) !\left(n \gamma_{1}^{*} \sigma_{0, E}^{2}+\sigma_{0,1}^{2}\right)} e^{-\frac{n\left(\gamma_{1}^{*}-1\right)}{\Lambda^{2} \alpha_{1} \rho_{0} \sigma_{0,1}^{2}}} \\
& \times\left(\sum_{n=0}^{\sum_{n=0}^{N} \frac{(-1)^{s} \Lambda^{2} \alpha_{2} N !}{n !(N-n) ! \rho_{0} \sigma_{0, E}^{2}} \int_{0}^{\infty} \frac{1}{\mu^{2}} e^{-\left(\frac{n \Omega}{\left(\Delta^{2} \alpha_{2}-\Lambda^{2} \alpha_{1} \Omega\right) \rho_{0} \sigma_{0, i}^{2}}+\frac{x}{\mu^{2}}\right)} d x} K_{K_{i}(i=1)}\right. \\
& \left.+e^{-\frac{\varpi}{\left(\alpha_{2}-\alpha_{1} \varpi\right) \rho_{0} \sigma_{0, E}^{2}}}\right)
\end{aligned}
$$

Using CDF (72) and Gaussian-Chebyshev quadrature method, $K_{i}$ for $i=\{1,2\}$ can be obtained and expressed as follows:

$$
\begin{aligned}
K_{i}= & \sum_{n=0}^{N=S U} \frac{(-1)^{n} \Delta^{2} \alpha_{2} N !}{n !(N-n) ! \rho_{0} \sigma_{0, E}^{2}} \\
& \times \int_{0}^{\varpi} \frac{1}{\left(\Delta^{2} \alpha_{2}-\Lambda^{2} \alpha_{1} x\right)^{2}} \\
& e^{-\left(\frac{n\left(\gamma_{2}^{*}(1+x)-1\right)}{\left(\Delta^{2} \alpha_{2}-\Lambda^{2} \alpha_{1}\left(\gamma_{2}^{*}(1+x)-1\right)\right) \rho_{0} \sigma_{0, i}^{2}}+\frac{x}{\left(\Delta^{2} \alpha_{2}-\Lambda^{2} \alpha_{1} x\right)^{2}}\right)} d x \\
= & \frac{\varpi \pi \Delta^{2} \alpha_{2} N !}{2 W \rho_{0} \sigma_{0, E}^{2}} \sum_{n=0}^{N} \sum_{w=1}^{W} \frac{(-1)^{n} \sqrt{1-\lambda^{2}} e^{-\Phi_{i}}}{n !(N-n) !\left(\Delta^{2} \alpha_{2}-\Lambda^{2} \alpha_{1} \beta\right)^{2}} .
\end{aligned}
$$

By substituting (73) into (72), we obtained the closed form of the SOP at $U_{1}$ over the MIMO scheme as shown in(46). From (73), we can also obtain the closed form of the SOP at $U_{2}$ over the MIMO scheme as shown in the (48) with outage conditions as shown in (47). 


\section{Declaration}

This study was conducted by the first author and assessed by the second author, supported by the use of Matlab software without any duplication or theft of results from previous studies. If any results bear a resemblance to another study, it was not our intention and is purely coincidental. To the best of our knowledge, the results were extracted from the Matlab application clearly and accurately.

\section{Abbreviations}

5G: Fifth generation network; BS: Base station; CDF: Cumulative distribution function; MIMO: Cumulative distribution function; MISO:

Multi-input-single-output; NOMA: Non-orthogonal multiple access; NOP:

Non-secrecy outage probability; PA: Power allocation; PDF: Probability density function; PLS: Physical layer security; SIC: Successive interference cancellation; SINRs: Signal-to-interference-plus-noise-ratios; SISO:

Single-input-single-output; SNRs: Signal-to-noise-ratios; SOP: Secrecy outage probability; TAS: Transmit antenna selection

\section{Acknowledgments}

We would especially like to thank the editors and anonymous reviewers for their helpful comments to improve this paper.

\section{Authors' contributions}

TNT is the first author who proposed the main concept, analysed and simulated the system and presented the final manuscript. MV is the second author. He is experienced in wireless communication research. He conducted a review and provide the first author with useful commentary. Both authors have read and approved the final manuscript.

\section{Authors' information}

Thanh-Nam TRAN (ORCID: 0000-0002-7065-7951) was born on Oct. 15, 1988 in the Vinh Long province, Vietnam. He received his M.Sc. from the Military Technical Academy (MTA) in 2014. He works and lectures at the Faculty of Electronics and Telecommunications at Sai Gon University, Vietnam. He is a member of the Wireless Communication Research Group and Faculty of Electrical and Electronics Engineering at Ton Duc Thang University. He is currently pursuing his Ph.D. in communications technology at VSB - Technical University of Ostrava, Czech Republic. He received Prof. Miroslav Voznak as a supervisor. His major interests are NOMA, energy harvesting (EH), cognitive radio (CR) and physical layer security (PLS). He has significant skill in $\mathrm{C}++$, python, and Matlab programming.

Miroslav VOZNAK (ORCID: 0000-0001-5135-7980) received his Ph.D. in telecommunications from the Faculty of Electrical Engineering and Computer Science at VSB - Technical University of Ostrava and completed his habilitations in 2002 and 2009. He was appointed Full Professor in 2017 in Electronics and Communications Technologies. He is an IEEE senior member and has served as a member on editorial boards for several publications, such as the Journal of Communications and the Advances in Electrical and Electronic Engineering Journal. His research interests focus generally on information and communications technology, particularly on quality of service and experience, network security, wireless networks and, in the last few years, big data analytics.

\section{Funding}

The research leading to these results received funding from the Czech Ministry of Education, Youth and Sports under grant No. SP2019/41 conducted at VSB Technical University of Ostrava.

\section{Availability of data and materials}

Data sharing is not applicable to this article as no datasets were generated or analysed during the study.

\section{Competing interests}

The authors declare no conflict of interest in producing this article.

Received: 4 June 2019 Accepted: 30 October 2019

Published online: 09 January 2020

\section{References}

1. Z. Ding, et al., Application of non-orthogonal multiple access in LTE and 5G networks. IEEE Commun. Mag. 55(2), 185-191 (2017). https://doi.org/ $10.1109 /$ mcom. $2017.1500657 \mathrm{~cm}$
2. L. Dai, B. Wang, Y. Yuan, S. C. I. Han, Z. Wang, Non-orthogonal multiple access for $5 \mathrm{G}$ : solutions, challenges, opportunities, and future research trends. IEEE Commun. Mag. 53(9), 74-81 (2015). https://doi.org/10.1109/ mcom.2015.7263349

3. Y. Saito, A. Benjebbour, Y. Kishiyama, T. Nakamura, in 2015 IEEE 81st Vehicular Technology Conference (VTC Spring). System-level performance of downlink non-orthogonal multiple access (NOMA) under various environments, (Glasgow, 2015), pp. 1-5. https://doi.org/10.1109/ VTCSpring.2015.7146120

4. Z. Ding, P. Fan, H. Poor, Impact of user pairing on $5 \mathrm{G}$ non-orthogonal multiple-access downlink transmissions. IEEE Trans. Veh. Technol. 65(8), 6010-6023 (2016). https://doi.org/10.1109/tvt.2015.2480766

5. Y. Liu, G. Pan, H. Zhang, M. Song, On the capacity comparison between MIMO-NOMA and MIMO-OMA. IEEE Access. 4, 2123-2129 (2016). https:// doi.org/10.1109/access.2016.2563462

6. Z. Ding, Z. Yang, P. Fan, H. Poor, On the performance of non-orthogonal multiple access in $5 \mathrm{G}$ systems with randomly deployed users. IEEE Signal Process. Lett. 21(12), 1501-1505 (2014). https://doi.org/10.1109//sp.2014. 2343971

7. S. Timotheou, I. Krikidis, Fairness for Non-Orthogonal Multiple Access in 5G Systems. IEEE Signal Process. Lett. 22(10), 1647-1651 (2015). https:// doi.org/10.1109//sp.2015.2417119

8. J. Cui, Z. Ding, P. Fan, A novel power allocation scheme under outage constraints in NOMA systems. IEEE Signal Process. Lett. 23(9), 1226-1230 (2016). https://doi.org/10.1109/lsp.2016.2591561

9. Z. Yang, Z. Ding, P. Fan, N. Al-Dhahir, A general power allocation scheme to guarantee quality of service in downlink and uplink NOMA systems. IEEE Trans. Wirel. Commun. 15(11), 7244-7257 (2016). https://doi.org/10. 1109/twc.2016.2599521

10. S. Islam, N. Avazov, O. Dobre, K. Kwak, Power-domain non-orthogonal multiple access (NOMA) in $5 \mathrm{G}$ systems: potentials and challenges. IEEE Commun. Surv. Tutor. 19(2), 721-742 (2017). https://doi.org/10.1109/ comst.2016.2621116

11. K. Higuchi, A. Benjebbour, Non-orthogonal multiple access (NOMA) with successive interference cancellation for future radio access. IEICE Trans. Commun. 98(3), 403-414 (2015). https://doi.org/10.1587/transcom.e98.b. 403

12. K. Higuchi, Y. Kishiyama, Non-orthogonal multiple access using intra-beam superposition coding and successive interference cancellation for cellular MIMO downlink. IEICE Trans. Commun. 98(9), 1888-1895 (2015). https://doi.org/10.1587/transcom.e98.b.1888

13. Z. Zhang, Z. Ma, M. Xiao, Z. Ding, P. Fan, Full-duplex device-to-device aided cooperative non-orthogonal multiple access. IEEE Trans. Veh. Technol., 1-1 (2016). https://doi.org/10.1109/tvt.2016.2600102

14. T.-N. Tran, M. Voznak, Multi-points cooperative relay in NOMA system with $\mathrm{N}-1$ DF relaying nodes in $\mathrm{HD} / \mathrm{FD}$ mode for $\mathrm{N}$ user equipments with energy harvesting. Electronics. 8(2), 167 (2019). https://doi.org/10.3390/ electronics8020167

15. Y. Liu, Z. Ding, M. Elkashlan, H. Poor, Cooperative non-orthogonal multiple access with simultaneous wireless information and power transfer. IEEE J. Sel. Areas Commun. 34(4), 938-953 (2016). https://doi.org/10.1109/jsac. 2016.2549378

16. J. Men, J. Ge, C. Zhang, Performance analysis of non-orthogonal multiple access for relaying networks over Nakagami-m fading channels. IEEE Trans. Veh. Technol. 66(2), 1200-1208 (2017). https://doi.org/10.1109/TVT. 2016.2555399

17. C. Zhong, Z. Zhang, Non-orthogonal multiple access with cooperative full-duplex relaying. IEEE Commun. Lett. 20(12), 2478-2481 (2016). https://doi.org/10.1109/lcomm.2016.2611500

18. Y. Liu, Z. Ding, M. Elkashlan, J. Yuan, Non-orthogonal multiple access in large-scale underlay cognitive radio networks. IEEE Trans. Veh. Technol. 65(12), 10152-10157 (2016). https://doi.org/10.1109/tvt.2016.2524694

19. T.-N. Tran, D. Do, M. Voznak, On outage probability and throughput performance of cognitive radio inspired NOMA relay system. Adv. Electr. Electron. Eng. 16(4) (2018). https://doi.org/10.15598/aeee.v16i4.2801

20. F. Lu, M. Xu, L. Cheng, J. Wang, J. Zhang, G. Chang, Non-orthogonal multiple access with successive interference cancellation in millimeter-wave radio-over-fiber systems. J. Light. Technol. 34(17), 4179-4186 (2016). https://doi.org/10.1109/jlt.2016.2593665

21. H. Marshoud, V. Kapinas, G. Karagiannidis, S. Muhaidat, Non-orthogonal multiple access for visible light communications. IEEE Photon. Technol. Lett. 28(1), 51-54 (2016). https://doi.org/10.1109//pt.2015.2479600 
22. T.-N. Tran, M. Voznak, HD/FD and DF/AF with fixed-gain or variable-gain protocol switching mechanism over cooperative NOMA for green-wireless networks. Sensors. 19(8) (2019). https://doi.org/10.3390/ s19081845

23. Y. Liu, M. Elkashlan, Z. Ding, G. Karagiannidis, Fairness of user clustering in MIMO non-orthogonal multiple access systems. IEEE Commun. Lett., 1-1 (2016). https://doi.org/10.1109/lcomm.2016.2559459

24. Z. Ding, F. Adachi, H. Poor, The application of MIMO to non-orthogonal multiple access. IEEE Trans. Wirel. Commun. 15(1), 537-552 (2016) https://doi.org/10.1109/twc.2015.2475746

25. Y. Yu, H. Chen, Y. Li, Z. Ding, L. Song, B. Vucetic, Antenna selection for MIMO nonorthogonal multiple access systems. IEEE Trans. Veh. Technol. 67(4), 3158-3171 (2018). https://doi.org/10.1109/tvt.2017.2777540

26. J. Men, J. Ge, Non-orthogonal multiple access for multiple-antenna relaying networks. IEEE Commun. Lett. 19(10), 1686-1689 (2015). https:// doi.org/10.1109/lcomm.2015.2472006

27. W. Han, J. Ge, J. Men, Performance analysis for NOMA energy harvesting relaying networks with transmit antenna selection and maximal-ratio combining over Nakagami-m fading. IET Commun. 10(18), 2687-2693 (2016). https://doi.org/10.1049/iet-com.2016.0630

28. X. Liu, X. Wang, in 2016 IEEE 83rd Vehicular Technology Conference (VTC Spring). Efficient antenna selection and user scheduling in $5 \mathrm{G}$ massive MIMO-NOMA system, (Nanjing, 2016), pp. 1-5. https://doi.org/10.1109/ VTCSpring.2016.7504208

29. M. Bloch, J. Barros, M. Rodrigues, S. McLaughlin, Wireless information-theoretic security. IEEE Trans. Inf. Theory. 54(6), 2515-2534 (2008). https://doi.org/10.1109/tit.2008.921908

30. Y. Zhang, H. Wang, Q. Yang, Z. Ding, Secrecy sum rate maximization in non-orthogonal multiple access. IEEE Commun. Lett. 20(5), 930-933 (2016). https://doi.org/10.1109/lcomm.2016.2539162

31. Z. Qin, Y. Liu, Z. Ding, Y. Gao, M. Elkashlan, in 2016 IEEE International Conference on Communications (ICC). Physical layer security for $5 \mathrm{G}$ non-orthogonal multiple access in large-scale networks, (Kuala Lumpur, 2016), pp. 1-6. https://doi.org/10.1109/ICC.2016.7510755

32. Y. Liu, Z. Qin, M. Elkashlan, Y. Gao, F. Lajos Hanzo, Enhancing the physical layer security of non-orthogonal multiple access in largescale networks. IEEE Trans. Wirel. Commun. 16(3), 1656-1672 (2017). https://doi.org/10. 1109/TWC.2017.2650987

33. J. Zhu, Y. Zou, G. Wang, Y.-D. Yao, G. K. Karagiannidis, On secrecy performance of antenna-selection-aided MIMO systems against eavesdropping. IEEE Trans. Veh. Technol. 65(1), 214-225 (2016). https:// doi.org/10.1109/TVT.2015.2397195

34. H. Lei, C. Gao, I. S. Ansari, Y. Guo, Y. Zou, G. Pan, K. Qaraqe, Secrecy outage performance of transmit antenna selection for MIMO underlay cognitive radio systems over Nakagami-m channels. IEEE Trans. Veh. Technol. 66(3), 2237-2250 (2017). https://doi.org/10.1109/TVT.2016.2574315

35. H. Lei, et al., On secure NOMA systems with transmit antenna selection schemes. IEEE Access. 5, 17450-17464 (2017). https://doi.org/10.1109/ access.2017.2737330

36. B. Li, X. Qi, K. Huang, Z. Fei, F. Zhou, R. Hu, Security-reliability tradeoff analysis for cooperative NOMA in cognitive radio networks. IEEE Trans. Commun. 67(1), 83-96 (2019). https://doi.org/10.1109/tcomm.2018. 2873690

37. F. Zhou, Z. Chu, H. Sun, R. Hu, L. Hanzo, Artificial noise aided secure cognitive beamforming for cooperative MISO-NOMA using SWIPT. IEEE J. Sel. Areas Commun. 36(4), 918-931 (2018). https://doi.org/10.1109/jsac. 2018.2824622

38. F. Zhou, Z. Chu, Y. Wu, N. Al-Dhahir, P. Xiao, in 2018 IEEE International Conference on Communications Workshops (ICC Workshops). Enhancing PHY Security of MISO NOMA SWIPT Systems with a Practical Non-Linear EH Model, (2018). https://doi.org/10.1109/iccw.2018

39. F. Jameel, S. Wyne, G. Kaddoum, T. Duong, A comprehensive survey on cooperative relaying and jamming strategies for physical layer security. IEEE Commun. Surv. Tutor. 21(3), 2734-2771 (2019). https://doi.org/10. 1109/comst.2018.2865607

40. L. Kong, S. Vuppala, G. Kaddoum, Secrecy Analysis of Random MIMO Wireless Networks Over $\alpha-\mu$ Fading Channels. IEEE Transactions on Vehicular Technology. 67(12), 11654-11666 (2018). https://doi.org/10. 1109/tvt.2018.2872884
41. D. Tran, H. Tran, D. Ha, G. Kaddoum, Secure transmit antenna selection protocol for MIMO NOMA networks over Nakagami-m channels. IEEE Syst. J., 1-12 (2019). https://doi.org/10.1109/jsyst.2019.2900090

42. Y. Feng, S. Yan, Z. Yang, Secure transmission to the strong user in non-orthogonal multiple access. IEEE Commun. Lett. 22(12), 2623-2626 (2018). https://doi.org/10.1109/lcomm.2018.2877320

43. Y. Feng, S. Yan, C. Liu, Z. Yang, N. Yang, Two-stage relay selection for enhancing physical layer security in non-orthogonal multiple access. IEEE Trans. Inf. Forensic. Secur. 14(6), 1670-1683 (2019). https://doi.org/10. 1109/tifs.2018.2883273

44. Z. Yang, Z. Ding, P. Fan, N. Al-Dhahir, The impact of power allocation on cooperative non-orthogonal multiple access networks with SWIPT. IEEE Trans. Wirel. Commun. 16(7), 4332-4343 (2017). https://doi.org/10.1109/ twc.2017.2697380

45. I. Abu Mahady, E. Bedeer, S. Ikki, H. Yanikomeroglu, Sum-rate maximization of NOMA systems under imperfect successive interference cancellation. IEEE Commun. Lett. 23(3), 474-477 (2019). https://doi.org/ 10.1109/lcomm.2019.2893195

46. J. Xu, L. Liu, R. Zhang, Multiuser MISO beamforming for simultaneous wireless information and power transfer. IEEE Trans. Signal Process. 62(18), 4798-4810 (2014). https://doi.org/10.1109/tsp.2014.2340817

47. X. Yue, Y. Liu, S. Kang, A. Nallanathan, Performance analysis of NOMA with fixed gain relaying over Nakagami- $m$ fading channels. IEEE Access. 5, 5445-5454 (2017). https://doi.org/10.1109/access.2017.2677504. Accessed 26 Aug 2019

48. S. Arzykulov, T. Tsiftsis, G. Nauryzbayev, M. Abdallah, Outage performance of cooperative underlay CR-NOMA with imperfect CSI. IEEE Commun. Lett. 23(1), 176-179 (2019). https://doi.org/10.1109/LCOMM.2018.2878730

\section{Publisher's Note}

Springer Nature remains neutral with regard to jurisdictional claims in published maps and institutional affiliations.

\section{Submit your manuscript to a SpringerOpen ${ }^{\circ}$ journal and benefit from:}

- Convenient online submission

Rigorous peer review

- Open access: articles freely available online

- High visibility within the field

- Retaining the copyright to your article

Submit your next manuscript at $>$ springeropen.com 